\title{
Optical simulations for design, alignment, and performance prediction of silicon pore optics for the ATHENA x-ray telescope
}

Spiga, D.; Della Monica Ferreira, Desiree; Shortt, B.; Bavdaz, M.; Bergback Knudsen, E.; Bergbäck Knudsen, Erik; Bianucci, G.; Christensen, Finn Erland; Civitani, M.; Collon, M.

Total number of authors:

21

Published in:

Optics for EUV, X-Ray, and Gamma-Ray Astronomy VIII

Link to article, DOI:

$10.1117 / 12.2274905$

Publication date:

2017

Document Version

Publisher's PDF, also known as Version of record

Link back to DTU Orbit

Citation (APA):

Spiga, D., Della Monica Ferreira, D., Shortt, B., Bavdaz, M., Bergback Knudsen, E., Bergbäck Knudsen, E., Bianucci, G., Christensen, F. E., Civitani, M., Collon, M., Conconi, P., Fransen, S., Marioni, F., Massahi, S., Pareschi, G., Salmaso, B., Jegers, A. S., Tayabaly, K., Valsecchi, G., ... Wille, E. (2017). Optical simulations for design, alignment, and performance prediction of silicon pore optics for the ATHENA x-ray telescope. In S. L. O'Dell, \& G. Pareschi (Eds.), Optics for EUV, X-Ray, and Gamma-Ray Astronomy VIII (Vol. 10399). [103990H] SPIE - International Society for Optical Engineering. Proceedings of SPIE - The International Society for Optical Engineering https://doi.org/10.1117/12.2274905

\section{General rights}

Copyright and moral rights for the publications made accessible in the public portal are retained by the authors and/or other copyright owners and it is a condition of accessing publications that users recognise and abide by the legal requirements associated with these rights.

- Users may download and print one copy of any publication from the public portal for the purpose of private study or research.

- You may not further distribute the material or use it for any profit-making activity or commercial gain

- You may freely distribute the URL identifying the publication in the public portal 


\section{Optical simulations for design, alignment, and performance prediction of silicon pore optics for the ATHENA x-ray telescope (Conference Presentation)}

D. Spiga, D. Della Monica Ferreira, B. Shortt, M. Bavdaz, E. Bergback Knudsen, et al.

D. Spiga, D. Della Monica Ferreira, B. Shortt, M. Bavdaz, E. Bergback Knudsen, G. Bianucci, F. Christensen, M. Civitani, M. Collon, P. Conconi, S. Fransen, F. Marioni, S. Massahi, G. Pareschi, B. Salmaso, A. S. Jegers, K. Tayabaly, G. Valsecchi, N. Westergaard, E. Wille, "Optical simulations for design, alignment, and performance prediction of silicon pore optics for the ATHENA x-ray telescope (Conference Presentation)," Proc. SPIE 10399, Optics for EUV, X-Ray, and Gamma-Ray Astronomy VIII, 103990H (19 September 2017); doi: 10.1117/12.2274905

Event: SPIE Optical Engineering + Applications, 2017, San Diego, California, United States 


\title{
Optical simulations for design, alignment, and performance prediction of silicon pore optics for the ATHENA X-ray telescope
}

\author{
D. Spiga,${ }^{1}$ D. Della Monica Ferreira, ${ }^{2}$ B. Shortt, ${ }^{3}$ M. Bavdaz, ${ }^{3}$ E. Bergbäck Knudsen, ${ }^{4}$ \\ G. Bianucci, ${ }^{5}$ F. Christensen, ${ }^{2}$ M. Civitani,${ }^{1}$ M. Collon,${ }^{6}$ P. Conconi, ${ }^{1}$ S. Fransen,${ }^{3}$ \\ F. Marioni,${ }^{5}$ S. Massahi,,${ }^{2}$ G. Pareschi,${ }^{1}$ B. Salmaso, ${ }^{1}$ A. S Jegers, ${ }^{2}$ K. Tayabaly,${ }^{7}$ \\ G. Valsecchi, ${ }^{5}$ N. Westergaard, ${ }^{2}$ E. Wille ${ }^{3}$ \\ ${ }^{1} \mathrm{INAF}$ - Brera Astronomical Observatory, Via Bianchi 46, 23807, Merate (Italy) \\ ${ }^{2}$ DTU Space, Techn. Univ. of Denmark, Elektrovej, bygn. 327, 2800 Kgs. Lyngby (Denmark) \\ ${ }^{3}$ European Space Agency, ESTEC, Keplerlaan 1, 2201 AZ Noordwjik (The Netherlands) \\ ${ }^{4}$ DTU Fysik, Techn. Univ. of Denmark, Fysikvej, bygn. 311, 2800 Kgs. Lyngby (Denmark) \\ ${ }^{5}$ Media Lario s.r.l., loc. Pascolo, 23842 Bosisio Parini (Italy) \\ ${ }^{6}$ Cosine, Oosteinde 36, $2361 \mathrm{HE}$ Warmond (The Netherlands) \\ ${ }^{7}$ Politecnico di Milano, Via La Masa 1, 20156 Milano (Italy)
}

\begin{abstract}
The ATHENA X-ray observatory is a large-class ESA approved mission, with launch scheduled in 2028. The technology of silicon pore optics (SPO) was selected as baseline to assemble ATHENA's optic with hundreds of mirror modules, obtained by stacking wedged and ribbed silicon wafer plates onto silicon mandrels to form the Wolter-I configuration. In the current configuration, the optical assembly has a $3 \mathrm{~m}$ diameter and a $2 \mathrm{~m}^{2}$ effective area at $1 \mathrm{keV}$, with a required angular resolution of 5 arcsec. The angular resolution that can be achieved is chiefly the combination of i) the focal spot size determined by the pore diffraction, ii) the focus degradation caused by surface and profile errors, iii) the aberrations introduced by the misalignments between primary and secondary segments, iv) imperfections in the co-focality of the mirror modules in the optical assembly. A detailed simulation of these aspects is required in order to assess the fabrication and alignment tolerances; moreover, the achievable effective area and the angular resolution depend on the mirror module design. Therefore, guaranteeing these optical performances requires: a fast design tool to find the most performing solution in terms of mirror module geometry and population, and an accurate point spread function simulation from local metrology and positioning information. In this paper, we present the results of simulations in the framework of ESA-financed projects (SIMPOSiuM, ASPHEA, SPIRIT) to prepare the ATHENA X-ray telescope: we deal with a detailed description of diffractive effects in an SPO mirror module, show ray-tracing results including mirror module misalignments, study in detail diffractive effects in different configurations, and assess the focal spot correspondence in X-rays and in the UV light, an important aspect to perform the mirror module alignment and integration. We also include a proton tracing simulation through a magnetic diverter in Halbach array configuration.
\end{abstract}

Keywords: ATHENA, silicon pore optics, design, simulation, alignment, diffraction

\section{INTRODUCTION}

The ATHENA X-ray telescope, ${ }^{1}$ currently in the system study phase for the L2 launch slot in 2028, will be the largest X-ray observatory ever built. It will have a 5 arcsec angular resolution (half-energy width, HEW), and - as a baseline design - an effective area of $2 \mathrm{~m}^{2}$ at $1 \mathrm{keV}$ plus a $0.25 \mathrm{~m}^{2}$ at $6 \mathrm{keV}$. The technology of silicon pore optics (SPO) is developed at ESTEC since 2004 in order to manufacture lightweight, segmented, and focusing optics with the mentioned characteristics. SPO mirror modules (MMs, Fig. 1), currently manufactured at Cosine ${ }^{2}$ consist of stacks of double-side polished, wedged, and grooved silicon wafers. The grooved plates

e-mail: daniele.spiga@brera.inaf.it, phone: +39-02-72320427

Optics for EUV, X-Ray, and Gamma-Ray Astronomy VIII, edited by Stephen L. O'Dell, Giovanni Pareschi, Proc. of SPIE Vol. 10399, 103990H · (C) 2017 SPIE · CCC code: 0277-786X/17/\$18 · doi: 10.1117/12.2274905 
are coated with an $\mathrm{Ir} / \mathrm{B}_{4} \mathrm{C}$ (either bi- or multi-layer) deposited on their non-grooved surface, ${ }^{3}$ then stacked onto silicon mandrels that endow them with the required curvatures in both directions: in the longitudinal direction, the profile is designed to reproduce the Wolter-I geometry. ${ }^{4}$ The adhesion of plates is ensured by molecular interactions between the accurately polished surfaces when they come into contact: to this end, the surfaces where the ribs adhere to the plate underneath have to be kept coating-free using a photolithographic process. ${ }^{5}$ Each MM is obtained by assembling two double stacks in sequence, with pores aligned to have rays reflected twice on the coated silicon surfaces and focused onto the $12 \mathrm{~m}$-distant instrument plane. Finally, all the MMs have to be integrated into the complete mirror assembly of ATHENA, in a Wolter-Schwarzschild configuration. ${ }^{6}$

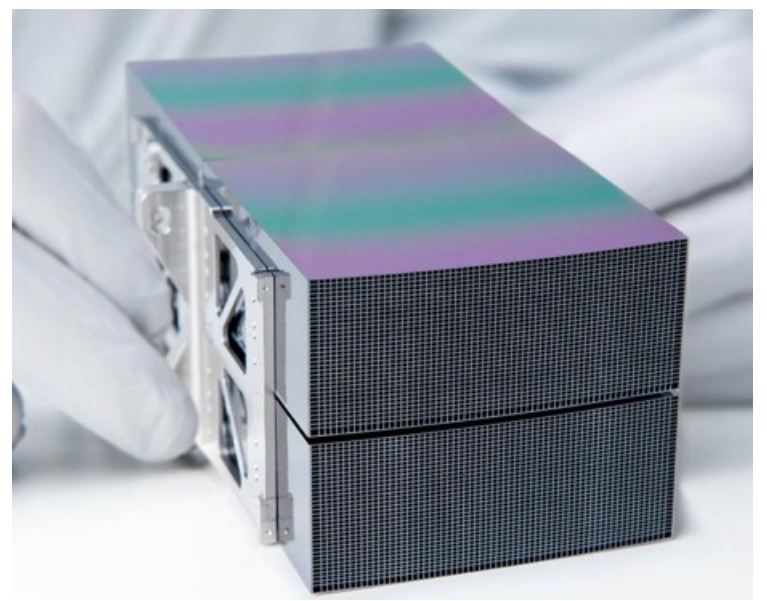

Figure 1. A Wolter-I SPO mirror module (image credits: ESA). A single mirror module consists of two Wolter-I double stacks with 34 plate pairs each, plus a functional plate pair to complete the stack. Typical pore pitch values are 1 to $3 \mathrm{~mm}$, while the pore height is $0.606 \mathrm{~mm}$. The mirror membrane is $0.17 \mathrm{~mm}$ thick, while the rib width can be constant (also $0.17 \mathrm{~mm}$ thick) or, for the inner mirror modules, variable along the plate width to ensure the radial stacking of ribs. ${ }^{7}$

Monocrystalline silicon has a very high thermal conductivity, which makes it an ideal material to minimize thermal gradients in space operation. Moreover, silicon wafers with high uniformity and smoothness are already available in the semiconductor market at an affordable cost, therefore the task of polishing a large mirror surface to sub-nanometer accuracy - classically, expensive and time-consuming - is immediately achieved. ${ }^{6}$ The stacking and profiling process of silicon plates, indeed, has proven to be very complex and its development required a considerable effort in order to improve the optical performances of SPO MMs, which are currently approaching the requirements for ATHENA. ${ }^{8}$ Other critical points in the development of the ATHENA optical system are for instance - the mirror design optimization, the determination of surface smoothness tolerances, the accurate alignment of hundreds of MMs to a common focus, ${ }^{9}$ the prediction of the achievable focusing properties, the stray light level assessment, the study of countermeasures for the charged particle background, ${ }^{10,11}$ and the final performance verification and calibration. ${ }^{12}$

A number of parallel studies, supported by ESA, are on their way to ensure the final performances of ATHENA. In the ASPHEA (Alignment of Silicon Pore Optics for High Energy Astrophysics) project, led by Media-Lario, we study the co-focal integration of mirror modules with the aid of an UV optical bench. In the Cosine-led SPIRIT (Silicon Pores for the Inner Radii of the ATHENA Telescope) project, we simulate the optical performances of mirror modules with suitable characteristics for the smallest radii of ATHENA (e.g., ribs with variable thickness along the plate width). Finally, in the context of the SIMPOSiuM (SIMulation of Pore OpticS and Modelling) project, a collaboration between INAF-OAB and DTU Space, we elaborate algorithms and pieces of software for the simulation of SPO performances. The early results of this collaboration have already been reported in a previous SPIE paper. ${ }^{13}$ In this paper, we show the results obtained in the second year of the SIMPOSiuM project, including the simulations in support to ASPHEA and SPIRIT.

An important activity, devoted to the optical design, has been developed this year. A baseline design for the ATHENA telescope has already been provided by ESA, ${ }^{14}$ but alternative designs may exist, e.g., privileging 
the field of view (e.g., wider rib spacing, shorter plate lengths) sometimes at the expense of some reduction of the on-axis area, or the adoption of polynomial profiles ${ }^{15}$ to limit off-axis aberrations. The most complex aspect, however, regards the optimization of the effective area (EA). Fortunately, it can be proven ${ }^{16,17}$ that the longitudinal curvature of mirrors that determines the angular resolution (e.g. Wolter-I vs. double cone) changes the effective area only by an amount $\sim L / 4 f$, where $L$ is the plate length and $f$ the focal length (Fig. 2). With the plate lengths used for ATHENA, we can even adopt a double cone geometry committing a $0.2 \%$ error in the effective area evaluation. The effective area is, in contrast, determined by the mirror module geometry parameters, such as the pore dimensions or the mirror plate length. Extending last year's work, ${ }^{13}$ in Sect. 2 we describe the way the on-axis and off-axis effective area depend on the pore size, the plate length, and so on. The effective area modeling allows us to promptly evaluate alternative designs in the mirror module assembly of ATHENA.

The performance prediction of a given optical design is a typical problem of geometric optics. If diffractive effects can be neglected at first order, the effective area and the point spread function (PSF) can be simulated using ray-tracing methods, possibly accounting for the X-ray scattering and for misalignments of MMs within the assembly or between the primary/secondary segment in each MM. In this project, the McXtrace package ${ }^{18}$ is being used to implement the complex structure of the ATHENA optics, assess the misalignments effects, and simulate the impact of stray light. Section 3 reports a result overview.

Diffractive effects in SPO MMs have been already detailed. ${ }^{13}$ In Sect. 4, we extend the 2D diffraction formalism to a variety of situations (source at finite distance, variable rib thickness, imperfect mirror profiles) that can be encountered in the optical characterization of an SPO MM. Simulations are carried out both in X-rays and UV light, in far-field approximation: we essentially find the same results of the 1D Fresnel diffraction theory, ${ }^{19,20}$ with the additional advantage to be able to assess the effect of azimuthal errors. In UV light, we show a comparison of simulation results with experimental images taken at the Media-Lario vertical optical bench (VOB): most important, we show that the position of the PSF centroid is fairly the same in X-rays and in UV light. This proves that the centroid position measured in the VOB is a reliable indicator of the expected focal spot location in X-rays, also for a complex structure such as an SPO's. This fact clearly validates the UV optical alignment of SPO MMs assemblies, as foreseen in the ASPHEA project. ${ }^{9}$

Surface microroughness (i.e. over a few microns lateral scales) cannot be directly included in the 2D diffraction figure computation, because the sampling of the aperture pupil would be too tight; we can therefore make use of the 1D diffraction theory, or even of the 1-st order scattering theory if the surface is sufficiently smooth. In the previous paper, ${ }^{13}$ we had measured the roughness PSD (power spectral density) on some coated silicon plates and evaluated the expected HEW degradation for increasing X-ray energy ${ }^{21}$ finding it within the high-energy specs for ATHENA $(<10$ arcsec below $6 \mathrm{keV})$. This time, we extend the simulation to a silicon plate that underwent the lithographic process needed for stacking plates; this sample exhibits residues of photoresist ${ }^{22}$ and we assess their impact on the expected HEW up to $6 \mathrm{keV}$ and beyond (Sect. 5).

The presence of charged particles - mostly, protons and electrons - in the orbital environment will expectedly be a major source of background for the ATHENA detectors. ${ }^{23}$ This issue has been addressed since Chandra and XMM times, ${ }^{24}$ but it is not completely understood to-date. In particular, the physical process responsible for grazing angle reflection of protons ${ }^{25}$ is not firmly determined, despite measurement campaigns using accelerators $^{26}$ and modeling works aimed at reproducing the background of solar protons in the XMM telescope. ${ }^{27}$ A known countermeasure can be a magnetic diverter, i.e. an arrangement of permanent magnets generating a magnetic field that deflects charged particles far from detectors. A possible magnetic diverter geometry for ATHENA is the Halbach array: unlike previous magnetic diverters located near the optics (e.g. for SWIFT-XRT or SIMBOL-X ${ }^{28,29}$ ), this equipment - already present in the ATHENA CDF, and under study also by Thales Alenia Space - generates a powerful magnetic field within a small volume, with minimal escape field and without obstructions of focused X-rays. For proper operation, the ring has to be compact, and therefore needs to be located quite close (a few meters) to the focal plane. In Sect. 6 , we simulate the magnetic field generated by a modeled Halbach array. ${ }^{30}$ Finally, we deal with some soft $(E<70 \mathrm{keV})$ proton tracing from the optics to the focal plane passing through the modeled magnetic field, and assessing the attenuation of the proton dose received by the WFI (wide field imager) detector. 


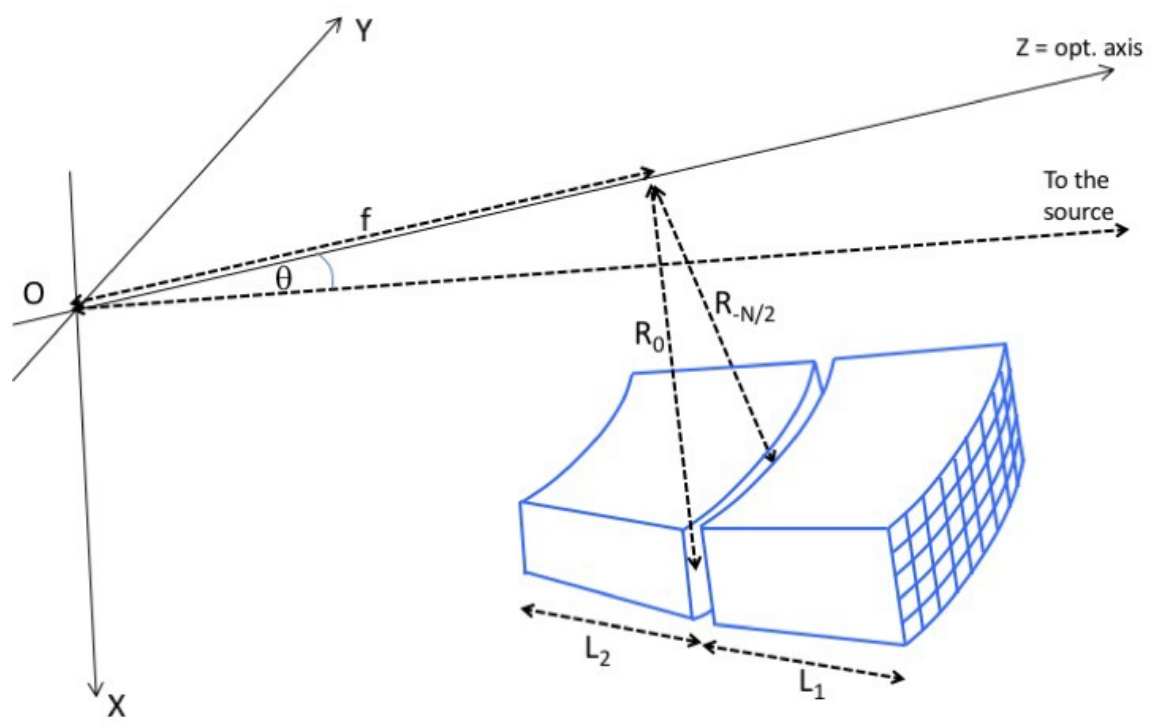

Figure 2. The model adopted to compute the effective area of an SPO mirror module. X-rays make two reflections in sequence onto a primary and a secondary stack. The X-ray source is assumed to be on the $x z$ plane, off-axis by an angle $\theta>0$. The plate radii $R_{n}$ increase throughout the stack for $n=-N / 2 \ldots+N / 2$. The central plate has radius $R_{0}$.

\section{EFFECTIVE AREA MODELING OF AN SPO MIRROR MODULE}

\subsection{Analytical description of the pore effective area}

In the previous paper ${ }^{13}$ we have drafted an analytical method to compute the effective area of a single pore in a generic polar angle $\varphi$ of the ATHENA mirror assembly, assuming a source at infinite distance on the positive side of the $x$-axis from which we measure $\varphi$, off-axis by an angle $\theta \geq 0$ (see Fig. 2). The pore width, measured along the azimuth, is $w$ and its height (along the radial direction) is $h$ (see Fig. 3). The mirrors plates are assumed to be $L_{1}$ long in the primary stack and $L_{2}$ in the secondary stack, as measured from the primary-secondary intersection plane, along the optical axis (Fig. 2). The plates are separated by two gaps $D_{1}$ and $D_{2}$ to the intersection plane, hence the material plate lengths are $L_{1}-D_{1}$ and $L_{2}-D_{2}$. In the current design and in this phase of the work, the plates have equal lengths and we can also suppose that the central pore in the stack has $D_{1}=D_{2}$. Therefore, we also have $L_{1}=L_{2}$ and denote with $L$ and $D$ their common values. We also assume all the silicon plates to be located at their nominal position (i.e. with distances to the optical axis matching the respective primary curvature radii). Finally, naming $\alpha_{0}$ the incidence angle on-axis and $\alpha_{1,2}$ the incidence angles off-axis on the primary and the secondary plate, we obtained the expression ${ }^{13}$

$$
A_{\text {pore }}(\lambda, \theta)=\underbrace{[w-2 \theta L|\sin \varphi|]_{\geq 0}}_{\text {azim. vign. }} \underbrace{\left[(L-D) \alpha_{0}-2 \theta L|\cos \varphi|\right]_{\geq 0}}_{\text {radial vignetting }} r_{\lambda}\left(\alpha_{1}\right) r_{\lambda}\left(\alpha_{2}\right),
$$

where $r_{\lambda}(\alpha)$ is the mirror reflectivity, computed using one of the standard methods, at the light wavelength $\lambda$ and the generic incidence angle $\alpha$. The real incidence angles ${ }^{16}$ are functions of $\alpha_{0}, \varphi, \theta$ :

$$
\begin{aligned}
& \alpha_{1}=\alpha_{0}-\theta \cos \varphi, \\
& \alpha_{2}=\alpha_{0}+\theta \cos \varphi,
\end{aligned}
$$

and the application of Eq. 1 turned out to be in excellent agreement with ray-tracing findings. The brackets []$_{\geq 0}$ mean that the enclosed expression shall be set to zero when negative.

We shall now find a generalized expression for the effective area of an MM, keeping the expression as flexible as possible in view of a design optimization. We refer to the MM aperture depicted in Fig. 3 with $N+1$ pores along the radius and $M+1$ along the azimuth, and we label the plates with the index $n=-N / 2, \ldots, 0, \ldots,+N / 2$, 


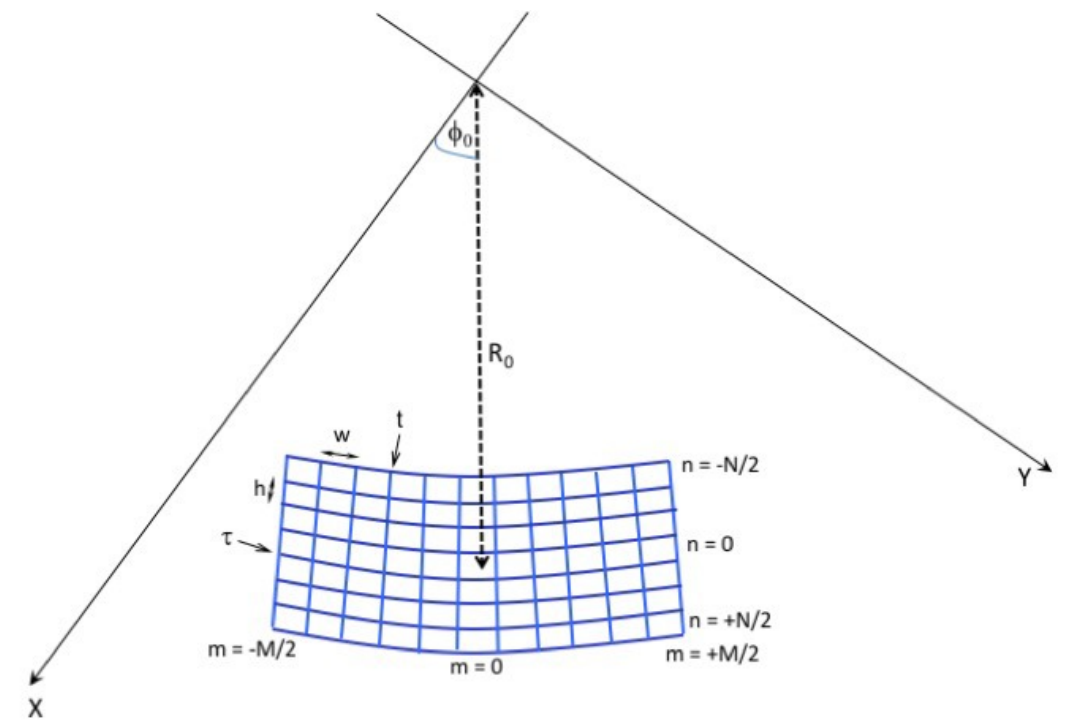

Figure 3. The aperture model for an SPO mirror module, seen from the positive $z$-axis. The source direction always remains in the $x z$ plane. The central pore of the stack has indices $(0,0)$ and is located by the polar coordinates $\left(R_{0}, \varphi_{0}\right)$. The other pores are located using the plate index $n=-N / 2, \ldots,+N / 2$ and the azimuthal index $m=-M / 2, \ldots,+M / 2$.

from the nearest plate to the optical axis outwards. Likewise, the azimuthal pore position is labeled by the index $m=-M / 2, \ldots, 0, \ldots,+M / 2$, in the direction of the increasing $\varphi$. To make the indices symmetric, we have considered here the case of a mirror module with an odd number of pores in the two directions, but the results can be adapted to an even number of pores simply replacing $N \rightarrow N-1$ and $M \rightarrow M-1$. In the following, we use $n$ and $m$ as superscripts for the incidence angles, and as subscripts for the other quantities.

Equation 1 was derived for an isolated pore. Some considerations apply in order to generalize this expression to an entire array of pores:

1. By construction, all the plates have a common intersection plane, even if the mirror assembly configuration is a Wolter-Schwarzschild's. Hence, the assumptions made ${ }^{13}$ to infer Eq. 1 remain valid also in this case.

2. The requirement for all the plates in the stack to have a common focus entails a gradual variation of $\alpha_{0}$ with $n$. The increase has to match the one of the plate radius near the intersection plane, i.e., $R_{n}=4 \alpha_{0}^{n} f$. This means that the primary plate's slope should be increased by $\Delta \alpha=(h+\tau) / 4 f$, and the secondary by $3 \Delta \alpha$, with respect to the inner neighbor plate. This is the wedge configuration referred to ${ }^{7}$ as $+1 /+3$, for which Eq. 1 was derived. Therefore, in this configuration the incidence angle on the $n$-th primary plate is $\alpha_{0}^{n}=\alpha_{0}^{0}+n \Delta \alpha$. For each plate, $\alpha_{1}^{n}+\alpha_{2}^{n}=2 \alpha_{0}^{n}$ is also the difference between the secondary and the primary slopes; hence, this difference needs to be increased by $2 \Delta \alpha$ at each stacking step.

3. In the standard design of ATHENA, the central plate length $L_{0}$ is tuned to exactly match the pore height when the source is on-axis, i.e., the incidence angle is the primary plate slope $\alpha_{0}^{0}$. In these conditions, $h=L_{0} \alpha_{0}^{0}$ and the inner edge of the central, primary plate is perfectly aligned to the outer edge of the adjacent plate with smaller radius (Fig. 4). This design - which we refer to as perfect nesting - obviously maximizes the on-axis effective area and the off-axis obstruction, which clearly helps to stop the stray light, ${ }^{31}$ but at the same time reduces the off-axis area, and consequently the field of view of the telescope. We can express the deviation from the perfect nesting condition using the filling factor parameter, expressed as the ratio $F F=R_{M}^{*} / R_{0}$, where $R_{0}$ is the central plate radius near the intersection plane of a reflecting plate surface and $R_{M}^{*}$ the backside's maximum radius of the next plate with smaller radius. The perfect nesting means that $F F=1$ (Fig. 4, left). If some additional space is left to mitigate off-axis obstruction (Fig. 4, right), we have $F F<1$. Solutions with $F F>1$ are seldom envisaged, ${ }^{32}$ as the effective area 
would even be obstructed on-axis. However, if the condition $F F=1$ is fulfilled in the central plate and the plate length is constant, the filling factor is not exactly 1 for the other plates, because the incidence angle changes. In the $+1 /+3$ wedge configuration, for example, we have $F F<1$ for the plates with $n<0$ and $F F>1$ for those with $n>0$.

4. Mirror module stacks are assembled from plates of constant dimensions, so we can in general assume $L-D$ constant in the mirror module. The stacks are tilted to form the correct angles $\left(\alpha_{0}\right.$ for the primary and $3 \alpha_{0}$ for the secondary) with the optical axis. Hence, $D_{1}$ and $D_{2}$ vary linearly in the stack,

$$
\begin{aligned}
& D_{1, n}=D_{0}-n(h+\tau) \alpha_{0}^{0}, \\
& D_{2, n}=D_{0}+3 n(h+\tau) \alpha_{0}^{0},
\end{aligned}
$$

where $D_{0}$ is the width of the central gaps, and $\alpha_{0}^{0}$ the on-axis incidence angle on the central plate. The small variation of $D \mathrm{~s}$ is irrelevant for the effective area if $F F \simeq 1$, because the obstruction caused by the gaps is completely dominated by other sources of obstruction. ${ }^{13}$
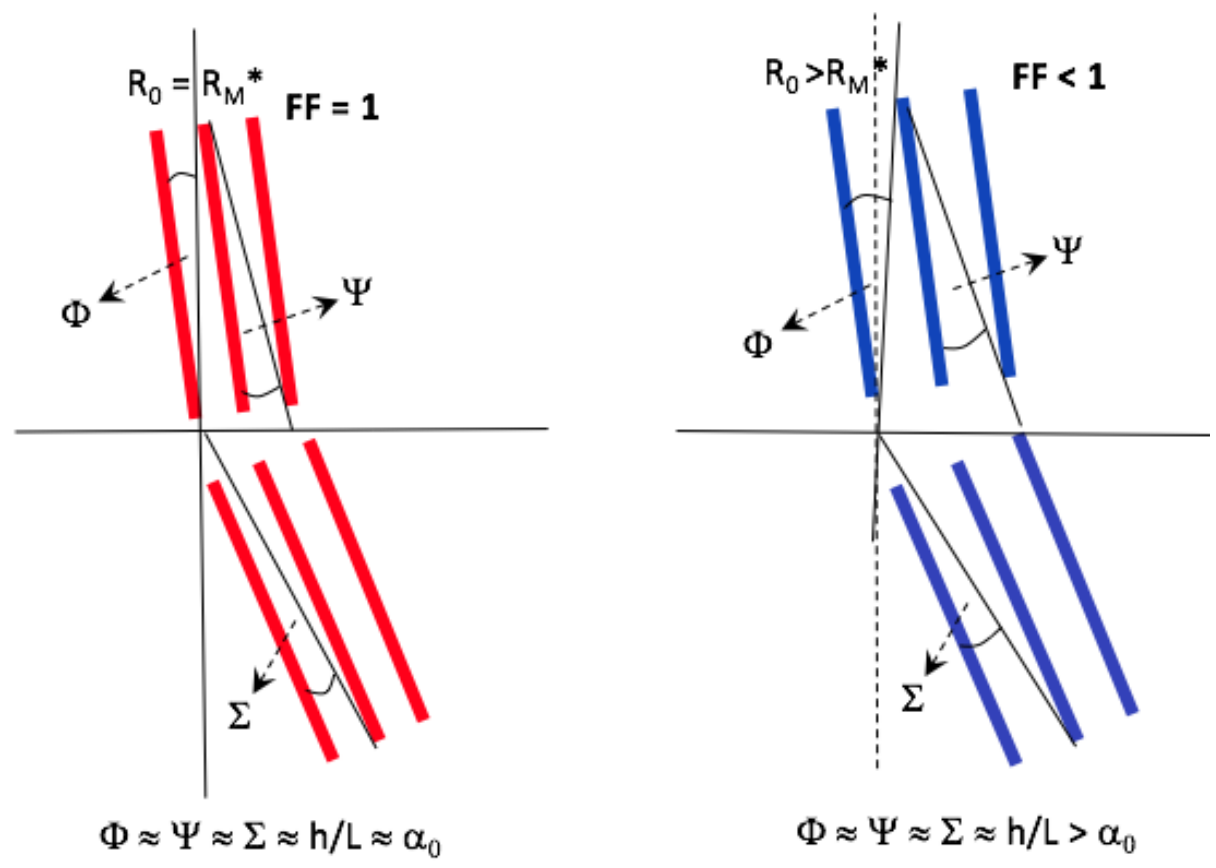

Figure 4. Obstruction parameters in an SPO MM stack. The $F F=1$ case (left) corresponds to the standard design for ATHENA. Solutions with $F F<1$ are detrimental for the on-axis effective area, but mitigate the off-axis obstruction.

5. The variation of $L$ with $n$ follows the same trend of $D$ if $L-D$ is a constant in the stack:

$$
\begin{aligned}
& L_{1, n}=L_{0}-n(h+\tau) \alpha_{0}^{0}, \\
& L_{2, n}=L_{0}+3 n(h+\tau) \alpha_{0}^{0},
\end{aligned}
$$

where $L_{0}$ is the length of the central plates. This variation can in principle have some effect in determining the variation of the obstruction parameters ${ }^{17}$ in the stack (Fig. 4). However, since $L \ll f$, we can assume that $\Phi \approx \Psi \approx \Sigma$. Moreover, since $h$ and $\tau$ are also constant in the MM stack, the obstruction parameter at the $n$-th pore becomes

$$
\Phi \approx \frac{h}{L_{0}}\left(1-\frac{3 n(h+\tau) \alpha_{0}}{L_{0}}\right) \approx \frac{h}{L_{0}}\left[1-3 n\left(\alpha_{0}\right)^{2}\right] \approx \frac{h}{L_{0}},
$$

and the factor multiplying $h / L_{0}$ differs from 1 by less than $2 \%$ for $|n|<20$ and the maximum value of $\alpha_{0}$ in ATHENA. We conclude that the variation of the obstruction parameter is a second-order effect. When 
$F F=1$, we have $h=\left(L_{0}-D_{0}\right) \alpha_{0}^{0}$, so we can rewrite Eq. 8 as $\Phi=\alpha_{0}^{0}\left(1-\xi_{0}\right)$, having set $\xi_{0}=D_{0} / L_{0}$. Equation 8 is valid in general, for $F F \leq 1$.

6. In the $-1 /+1$ wedge configuration, ${ }^{7}$ the increment of $2 \Delta \alpha$ is again the secondary-primary slope difference, but this time it is obtained decreasing the primary angle by $\Delta \alpha$ and increasing the secondary by the same amount, moving towards the outer plate pair. In this way, the same kind of wedged plates can be used for the two stacks: the only price to pay is that each plate "sees" the source off-axis by a small angle, but increasing with the distance from the central plate. This causes some effective area loss, because some rays either avoid the second reflection or get blocked, also on-axis. The relation between the primary and secondary reflection, which all the previous discussion ${ }^{13,16,17}$ was based on, is now altered and might seem questioned. However, a simple geometric construction (Fig. 5) shows that we can adapt the formalism adding a fictitious central divergence $\delta_{n}=-2 n \Delta \alpha$ of the source, variable with $n$ within the module. This change affects only the radial vignetting.
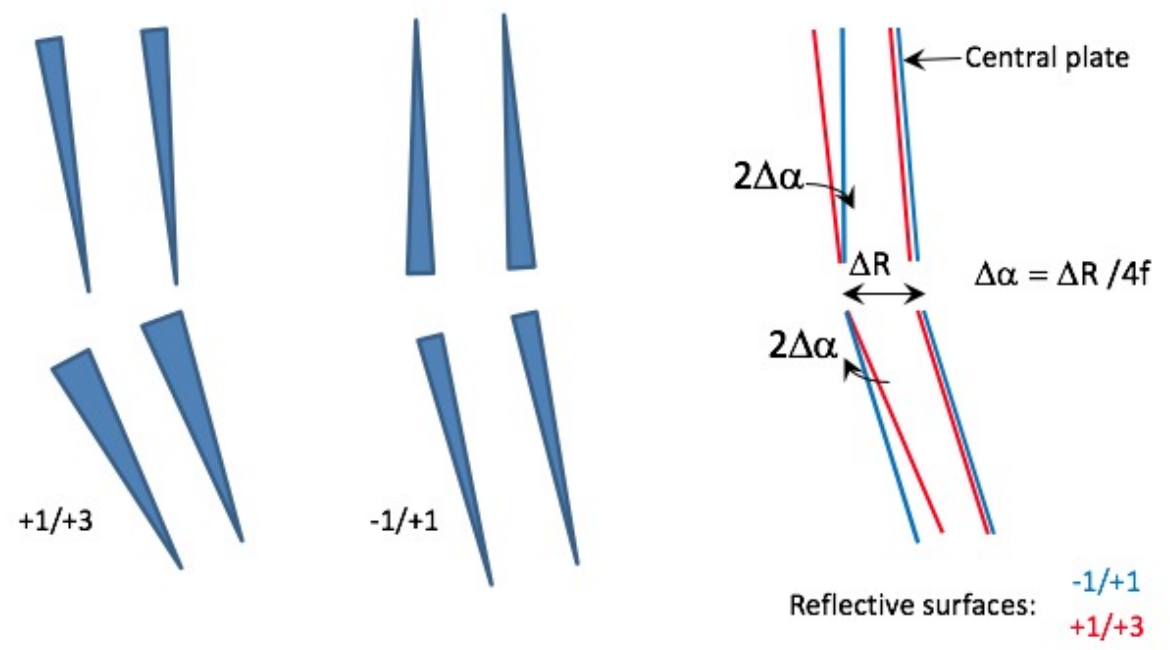

Figure 5. Different plate stacking in an SPO module, in radial section. Left: the $+1 /+3$ wedge configuration requires manufacturing two different kinds of wedged plates. Center: the $-1 /+1$ wedge configuration is obtained producing silicon plates with the same wedge angle. Right: with respect to the $+1 /+3$ wedge, the angle variation in the $-1 /+1$ wedge configuration introduces an apparent source divergence $\delta_{n}=-2 n \Delta \alpha$ for the $n$-th plate, counted from the central one.

We can thereby re-consider the more general expression for the effective area of the $n m$-th pore, ${ }^{13}$ always assuming $L_{1}=L_{2}=L_{0}$ and neglecting second order effects:

$$
A_{n m}(\lambda, \theta)=w V_{\mathrm{R}}\left(\varphi_{n m}\right) L_{0}\left[\min \left(\alpha_{1}^{n m}, \alpha_{2}^{n m}, \Phi\right)-\max \left(\alpha_{1}^{n m}-\Phi, \alpha_{2}^{n m}-\Phi, \xi_{0} \alpha_{1}^{n m}, \xi_{0} \alpha_{2}^{n m}\right)\right]_{\geq 0} r_{\lambda}\left(\alpha_{1}^{n m}\right) r_{\lambda}\left(\alpha_{2}^{n m}\right)
$$

where $V_{\mathrm{R}}\left(\varphi_{n m}\right)=1-2 \theta L_{0}\left|\sin \varphi_{n m}\right| / w$ is the azimuthal vignetting factor caused by the ribs, and $\Phi=h / L_{0}$ is the radial obstruction parameter, which is constant for all the pores in the module (Eq. 8). The angle $\varphi_{n m}$ is the polar angle of the $n m$-th pore, and clearly takes only on discrete values $\varphi_{n m}=\varphi_{0}+m \Delta \varphi_{n}$, where $\Delta \varphi_{n}=(w+t) / R_{n}$. The slope $\alpha_{0}$ and the incidence angles $\alpha_{1}, \alpha_{2}$ on the primary and secondary surface of the $n m$-th pore have the usual expressions, ${ }^{17}$ adapted to the current notation and including the fictitious off-axis angle related to the actual wedge configuration (Fig. 5):

$$
\begin{aligned}
\alpha_{0}^{n} & =\alpha_{0}^{n}+n \Delta \alpha_{\mathrm{R}}, \\
\alpha_{1}^{n m} & =\alpha_{0}^{n}-2 n \Delta \alpha_{\mathrm{w}}-\theta \cos \varphi_{n m}, \\
\alpha_{2}^{n m} & =\alpha_{0}^{n}+2 n \Delta \alpha_{\mathrm{w}}+\theta \cos \varphi_{n m},
\end{aligned}
$$


where we denoted with $\Delta \alpha_{\mathrm{w}}$ the slope increment in the adopted wedge configuration, and used the symbol $\Delta \alpha_{\mathrm{R}}=(h+\tau) / 4 f$ for the slope increment caused by the increasing radius. The central slope angle still follows the co-focality relation of MMs, $\alpha_{0}^{0}=R_{0} / 4 f$. In the $-1 /+1$ configuration we have $\Delta \alpha_{\mathrm{w}}=\Delta \alpha_{\mathrm{R}}$, while in the $+1 /+3$ one we have $\Delta \alpha_{\mathrm{w}}=0$. Equations 9 to 12 are generally applicable to any MM with $L_{1}=L_{2}$, and $F F \leq 1$, provided that the source is located at infinitely large distance.

We can easily extend the equations above to a situation where the source is at a finite distance $S$ from the intersection plane of the module (the case of on-ground sources, such as PANTER). ${ }^{12}$ With the source on-axis, the beam reaches the $n$-th plate with a divergence angle $\delta_{n}=R_{n} / S$ that introduces a further off-axis term. The incidence angles change as follows: ${ }^{16}$

$$
\begin{aligned}
& \alpha_{1}^{n m}=\alpha_{0}^{n}+\delta_{n}-2 n \Delta \alpha_{\mathrm{w}}-\theta \cos \varphi_{n m}, \\
& \alpha_{2}^{n m}=\alpha_{0}^{n}-\delta_{n}+2 n \Delta \alpha_{\mathrm{w}}+\theta \cos \varphi_{n m},
\end{aligned}
$$

with the usual conventions that the incidence angles should be set to zero whenever negative. Equations 9, 10, 13, 14 can be used to compute the $n m$-th pore EA in a completely generic configuration.

We conclude this paragraph pointing out that a stray light analytical modeling can in principle follow the same guidelines, as the expressions for the radial vignetting coefficients for stray light are known from previous works. ${ }^{31}$ However, the azimuthal vignetting factor $V_{R}$ caused by the ribs is more complicated to model for single reflections and will therefore be analyzed in a subsequent development.

\subsection{Application to the baseline configuration of mirror modules}

\subsubsection{Source at infinite distance}

We now apply Eq. 9 to the baseline configuration for ATHENA $(-1 /+1$ wedge and $F F=1)$, initially in the case of $S \rightarrow \infty$. For any $F F \leq 1$ and small $\xi_{0}$, we have $\min \left(\alpha_{1}^{n m}, \alpha_{2}^{n m}\right) \leq \alpha_{0}^{n} \leq \Phi$. Therefore, the terms appearing in Eq. 9 become

$$
\begin{aligned}
\min \left(\alpha_{1}^{n m}, \alpha_{2}^{n m}, \Phi\right) & =\alpha_{0}^{n}-\left|2 n \Delta \alpha_{\mathrm{R}}+\theta \cos \varphi_{n m}\right|, \\
\max \left(\alpha_{1}^{n m}-\Phi, \alpha_{2}^{n m}-\Phi, \xi_{0} \alpha_{1}^{n m}, \xi_{0} \alpha_{2}^{n m}\right) & =\max \left(\alpha_{0}^{n}-h / L_{0}+\left|2 n \Delta \alpha_{\mathrm{R}}+\theta \cos \varphi_{n m}\right|, \xi_{0} \alpha_{1}^{n m}, \xi_{0} \alpha_{2}^{n m}\right) .
\end{aligned}
$$

In Eq. 16, we could not remove the max operator because $\alpha_{0}^{n}-h / L_{0}<0$ if $F F<1$, while $\xi_{0} \alpha_{1}^{n m}$ and $\xi_{0} \alpha_{2}^{n m}$ are small, but always non-negative. This is, in fact, a case in which the gaps may have some impact on the effective area. Fortunately, the expression can be noticeably simplified in the perfect nesting case: substituting $h / L_{0}=\left(1-\xi_{0}\right) \alpha_{0}^{0}$ and Eq. 10 in Eq. 16, we turn it into

$$
\max \left(\alpha_{1}^{n m}-\Phi, \alpha_{2}^{n m}-\Phi, \xi_{0} \alpha_{1}^{n m}, \xi_{0} \alpha_{2}^{n m}\right)=\max \left(\xi_{0} \alpha_{0}^{0}+n \Delta \alpha_{\mathrm{R}}+\left|2 n \Delta \alpha_{\mathrm{R}}+\theta \cos \varphi_{n m}\right|, \xi_{0} \alpha_{1}^{n m}, \xi_{0} \alpha_{2}^{n m}\right) .
$$

The first term in the max operator prevails over the other two, if and only if

$$
\left(1-\xi_{0}\right)\left[n \Delta \alpha_{\mathrm{R}}+\left|2 n \Delta \alpha_{\mathrm{R}}+\theta \cos \varphi_{n m}\right|\right] \geq 0
$$

and since $\xi_{0} \ll 1$, this condition is always fulfilled if the other factor is non-negative. This definitely occurs if $\theta=0$; if $\theta>0$, the condition is also fulfilled for $n \geq 0$, while for $n<0$ we can violate Eq. 18 only if $\theta \cos \varphi_{n m}<-n \Delta \alpha_{\mathrm{R}}$. Due to the small value of $\Delta \alpha_{\mathrm{R}}=3.3 \mathrm{arcsec}$, and since in practice we have $|n|<20$, we conclude that the vignetting effect of the gaps overrides the other off-axis obstructions only for $\theta<\sim 1$ arcmin, i.e., in a negligibly small range of off-axis angles.

Therefore, taking the difference of Eq. 15 and Eq. 16 and substituting it into Eq. 9, we remain with

$$
A_{n m}(\lambda, \theta)=w V_{\mathrm{R}}\left(\varphi_{n m}\right) L_{0}\left[\alpha_{0}^{n}-\xi_{0} \alpha_{0}^{0}-n \Delta \alpha_{\mathrm{R}}-2\left|2 n \Delta \alpha_{\mathrm{R}}+\theta \cos \varphi_{n m}\right|\right]_{\geq 0} r_{\lambda}\left(\alpha_{1}^{n m}\right) r_{\lambda}\left(\alpha_{2}^{n m}\right) .
$$

Using now Eq. 10 and the relation $h=L_{0}\left(1-\xi_{0}\right) \alpha_{0}^{0}$, we obtain the expression of the effective area of the $n m$-th pore for the baseline configuration of the ATHENA mirror modules:

$$
A_{n m}(\lambda, \theta)=W_{n m} L_{n m} r_{\lambda}\left(\alpha_{1}^{n m}\right) r_{\lambda}\left(\alpha_{2}^{n m}\right),
$$


having set

$$
\begin{aligned}
W_{n m} & =\left[w-2 \theta L_{0}\left|\sin \left(\varphi_{0}+m \Delta \varphi_{n}\right)\right|\right]_{\geq 0}, \\
L_{n m} & =\left[h-2 L_{0}\left|2 n \Delta \alpha_{\mathrm{R}}+\theta \cos \left(\varphi_{0}+m \Delta \varphi_{n}\right)\right|\right]_{\geq 0} .
\end{aligned}
$$

and written the polar angle explicitly referred to the central polar angle $\varphi_{0}$. In this mirror module configuration, the expressions of the incidence angles (Eqs. 11 and 12) are needed only to compute the reflectivities in Eq. 20.

The effective area of a mirror module can be computed applying Eq. 20 over any range of $\lambda$, for any $\theta$ value, and summing over the $n, m$ indices. Because $r_{\lambda}(\alpha)$ is hard to model analytically, the sum should be computed numerically (Sect. 2.3). However, setting $r_{\lambda}(\alpha)=1$ and $\theta=0$, we can easily derive an expression for the total geometric area on-axis:

$$
A_{G}(0)=\sum_{-N / 2}^{N / 2} \sum_{-M / 2}^{M / 2} w\left[h-4 L_{0}|n| \Delta \alpha_{\mathrm{R}}\right]=(M+1) w\left[(N+1) h-\left(N^{2}+2 N\right) L_{0} \Delta \alpha_{\mathrm{R}}\right],
$$

where the second term in [] brackets expresses the diminished area in $-1 /+1$ wedge configuration. For a single pore $(N, M=0)$, the on-axis geometric area correctly reduces to $w h$. The fraction of lost area on-axis roughly scales as $N \Delta \alpha / \alpha_{0}^{0}$, therefore it can amount up to $9 \%$ for the inner radii of ATHENA.

\subsubsection{Source at finite distance}

Using Eq. 13 and 14, we can extend the results of the last paragraph to an effective area measurement setup with a source at distance $S<\infty$. Equation 20 remains valid, replacing the expressions for $W_{n m}$ and $L_{n m}$ by

$$
\begin{aligned}
W_{n m} & =\left[w-2 L_{0}\left|\theta \sin \left(\varphi_{0}+m \Delta \varphi_{n}\right)\right|\right]_{\geq 0}, \\
L_{n m} & =\left[h-2 L_{0}\left|2 n \Delta \alpha_{\mathrm{R}}+\theta \cos \left(\varphi_{0}+m \Delta \varphi_{n}\right)-\delta_{n}\right|\right]_{\geq 0},
\end{aligned}
$$

and the incidence angles, needed to compute the reflectivity values, are provided by Eqs. 13 and 14 .

The azimuthal vignetting term (Eq. 24) is unchanged with respect to the infinitely distant source case in Sect. 2.2.1, as expected. In fact, since the ribs are aligned radially, for $\theta=0$ there is no azimuthal vignetting regardless of the source distance finiteness. When $\theta>0$, the angle formed by the initial propagation vector $\left(\delta_{n} \cos \varphi-\theta, \delta_{n} \sin \varphi,-1\right)$ with the normal to a generic $\operatorname{rib}(\sin \varphi,-\cos \varphi)$ is always $\pi / 2-\theta \sin \varphi$, i.e., independent of $\delta_{n}$. If we now aim at minimizing the effective area loss of a MM due to the beam divergence, we can align the source to the central pore. This is equivalent to setting $\theta=\delta_{0}=R_{0} / S$ and $\varphi_{0}=0$ in the previous equations and clearly minimizes the radial vignetting. In fact, it is a typical configuration to calibrate the mirror module on-ground. The price to pay is, however, the reappearance of an azimuthal vignetting term $W_{n m}$ proportional to $\delta_{0}$.

In addition, a $\delta_{n}$ term has now appeared in Eq. 25, the radial vignetting term; we can write this angle as $\delta_{n}=\left(R_{0}+n(h+\tau)\right) / S$. In calibration mode $\left(\theta=\delta_{0}=R_{0} / S\right)$ and for a module in the off-axis plane $\left(\varphi_{0}=0\right)$ we can rewrite Eq. 25 in the form:

$$
L_{n m}\left(\delta_{0}\right)=\left[h-2 L_{0}\left|2 n \Delta \alpha_{\mathrm{R}}+R_{0} \frac{\cos (m \Delta \varphi)-1}{S}-n \frac{h+\tau}{S}\right|\right]_{\geq 0},
$$

where the last term in the absolute value expresses the residual divergence. Since the azimuthal aperture of a mirror module is usually small, $\cos (m \Delta \varphi) \approx 1$ and we can neglect the central term. Finally, in $-1 /+1$ wedge configuration, we can substitute $\Delta \alpha=(h+\tau) / 4 f$ and obtain an extremely simple formula for $L_{n m}$ :

$$
L_{n m}\left(\delta_{0}\right) \simeq\left[h-2 L_{0}(h+\tau)\left|n\left(\frac{1}{2 f}-\frac{1}{S}\right)\right|\right]_{\geq 0} .
$$

In the last equation, the second term in [] brackets is the residual vignetting caused by a combination of the beam divergence $1 / S$ and the wedge configuration $1 / 2 f$. As the two terms have different signs, we conclude that in MM calibration setup (and reasonably assuming $S>2 f$ ) the $-1 /+1$ wedge mitigates the radial vignetting caused by the residual divergence. 


\subsection{Full effective area computation}

An IDL code, implementing Eqs. 9, 10, 13, and 14, has been written aiming to rapidly compute the effective area of the mirror assembly from a design file. The computation can be performed on-axis, or off-axis, or for a divergent source, with either $-1 /+1$ or $+1 /+3$ wedge configuration, and for any filling factor $F F \leq 1$. The effective area expressions are always symmetric with respect to the $\mathrm{x}$-axis, so the computation can be limited to the upper two quadrants (Fig. 6). A typical computation time for the complete effective area is 2 min using a commercial computer (e.g., a MacBook equipped with a $2.4 \mathrm{GHz}$ processor and an $8 \mathrm{~GB}$ RAM), regardless of the off-axis angle. The computation time is short enough to allow us avoiding the approximation of sampling the effective area in a few pores per module, with the advantage to accurately account for vignetting variation effects, also within a $-1 /+1$ module. Finally, using an analytical computation, we are not affected by issues potentially connected to ray-tracing: non-uniform sampling of the aperture pupil, large computation time, and statistical uncertainties.
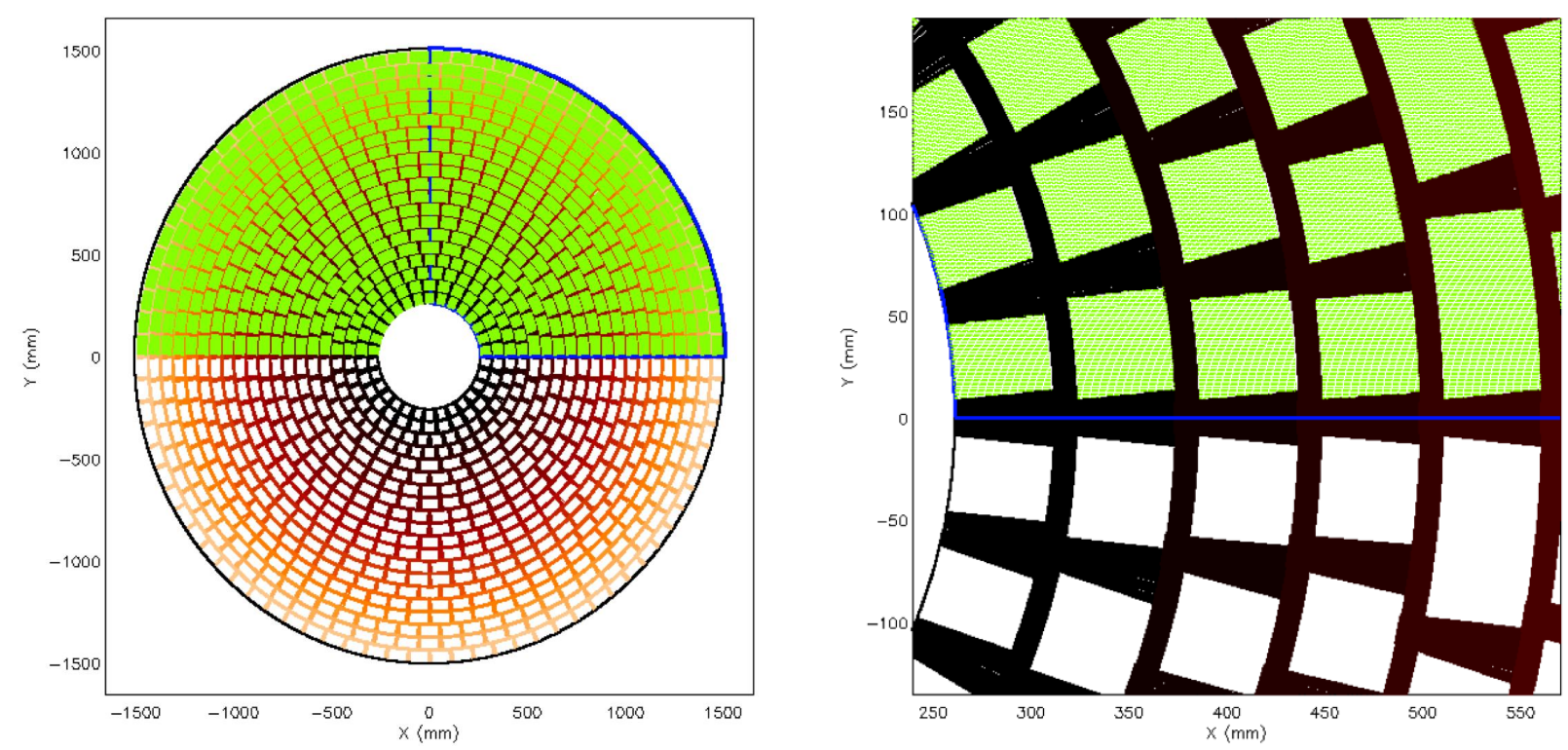

Figure 6. Left: the nominal MM structure of ATHENA. Due to the symmetry of the vignetting factors, the computation of the EA can be restricted to the upper two quadrants. Right: a central detail of the modeled aperture in the nominal design, showing the structure of the modules. Every pore counted in the EA computation is represented as a green dot.

The IDL code also includes the possibility to simulate the illumination of the ATHENA mirror assembly over sub-apertures in the shape of circular segments. This is useful to compute the expected effective area calibration of a small group of MMs at a time, in on-ground X-ray facilities. ${ }^{12}$ In this case, due to the finite source distance, the measured effective area would be reduced to $1 / 4$ of the nominal value if the source was placed on the optical axis. The beam divergence can partly be compensated for a single group of MMs by appropriately tilting the mirror assembly in such a way that the divergence angle is zeroed at the module center (Eq. 27). Anyway, the compensation is only approximate throughout the rest of the sub-aperture: this causes some effective area loss that can be minimized decreasing the aperture size, but at the expense of the number of sub-apertures to be tested. At this regard, the IDL tool for rapid EA computation can help one to find the best compromise between the two aspects. In the treatment hitherto described, the tilt (the off-axis angle $\theta$ ) is assumed to be in the $x z$ plane, therefore the sub-aperture should be centered on the $x$-axis; however, we have also extended the code to a more general situation in which a mirror module is tilted in any direction, therefore enabling the simulation of mirror module misalignments in both "pitch" and "yaw" directions.

The total computed effective area, for the standard design and an on-axis source at infinity, is displayed in Fig. 7, left, for the two possible wedge configurations: $+1 /+3$ (green line) and $-1 /+1$ (red line). As expected, there is a few percent EA decrease in the $-1 /+1$ configuration, because each off-center plate pair slightly sees the 
source off-axis. In Fig. 7, right, we have plotted the geometric area trend in concentric pore rows of increasing radius, as a function of the distance to the central axis, with each line segment representing a row of mirror modules. In the $+1 /+3$ configuration, the geometric area correctly increases in proportion with the incidence angle up to the central pore $(n<0)$, then saturates to the product of the pore area $w h$ times the (constant) number of pores in each row of MMs. This occurs because $F F<1$ when $n<0$ in each MM row, so the plate length is slightly too "short" to cover the pore height. Besides the increasing trend with the radius caused by the increasing number of pores within a quadrant, the $-1 /+1$ wedge setting matches the $+1 /+3$ only in the central plate of each MM row. All the others suffer from off-axis vignetting proportional to $|n|$, and this is responsible for the "cusped" trend in Fig. 7, right.
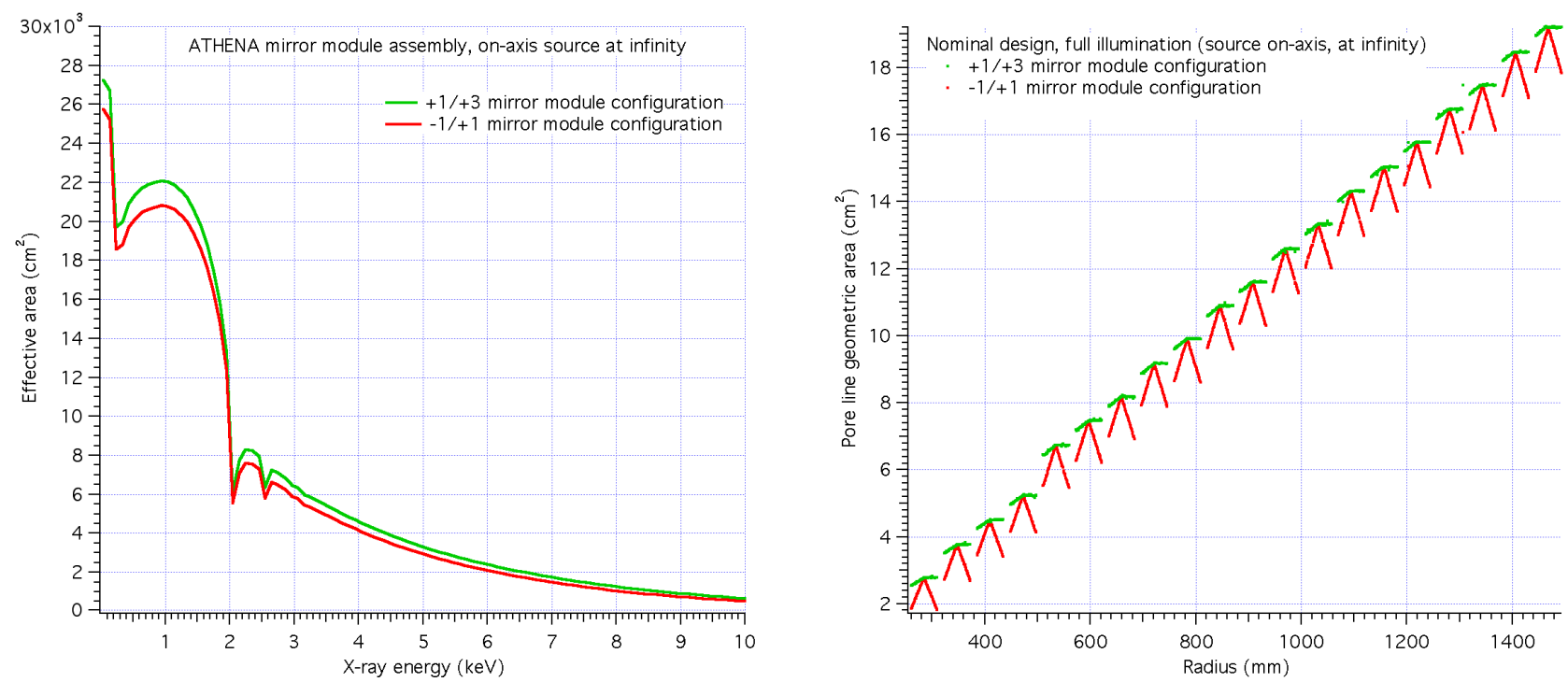

Figure 7. Left: the on-axis effective area of ATHENA, as computed with the simulation code in $+1 /+3$ and $-1 /+1$ wedge configuration, based on the algorithm described in Sect. 2.1. Right: the geometric area trend for all lines of pores, with the source on-axis and at infinity. We see the difference between the two wedge configurations caused by the variation of the apparent off-axis angle seen by the silicon plates.
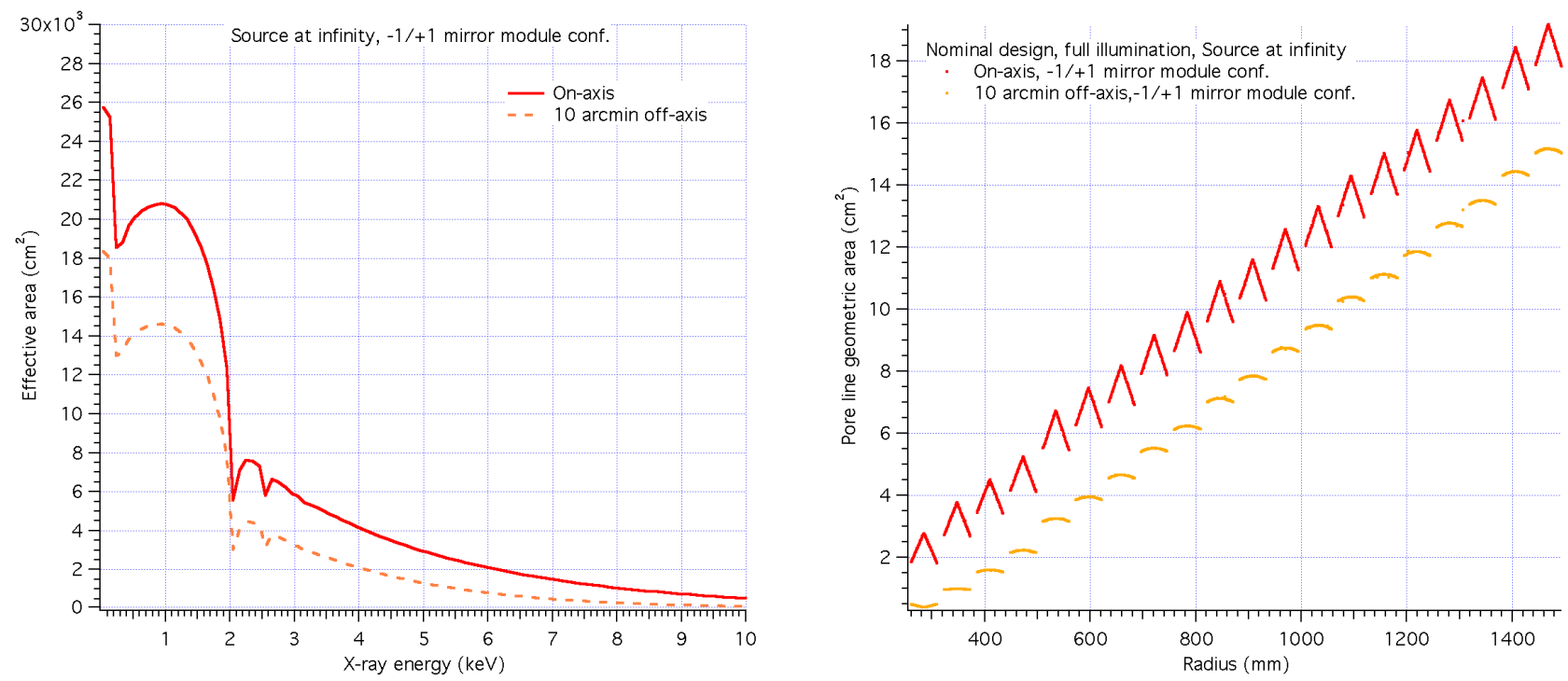

Figure 8. Left: The total EA of ATHENA, on-axis and 10 arcmin off-axis, using the nominal design and assuming the source at infinity. Right: the geometric area trend of the pore lines. 
The same formula (Eqs. 9) can obviously be used to treat the off-axis case. In Fig. 8 we show the computation results obtained by setting $\theta=10$ arcmin, always in the case of a source at infinity and $-1 /+1$ wedge setup. As expected, the EA is lower off-axis than on-axis (left) because of various sources of obstruction, with a geometric area trend rounded by the spread of incidence angles introduced off-axis (right).

\subsection{Alternative mirror assembly design}

In this section we start to explore some alternative designs for the mirror module assembly of ATHENA. Some constraints in the mirror module structure are dictated by the manufacturing process, and - at the time of writing - are not subject to change:

- all the plates in an MM stack have the same thickness, length, and width;

- all the plates in an MM primary stack has the same length and width of those in the secondary stack;

- all the pores have the same height and the same membrane thickness.


Figure 9. Left: representation of the nominal design for the ATHENA mirror assembly (also shown in Fig. 6, left). Right: alternative tessellation using mirror modules with an hypothetical width of $\sim 160 \mathrm{~mm}$.

Within these constraints, we can in principle devise any possible tessellation of the ATHENA optical aperture with mirror modules of variable height, width, length, with variable pore widths and heights. A simple IDL code finds the optimal population of mirror modules using the following input parameters:

1. the pore height $h$ and the membrane thickness $\tau$;

2. the pore width $w$ and the rib width $t$ (the latter is provisionally assumed constant);

3. the number of "petals" in the mirror module assembly;

4. the minimum and the maximum radius of the mirror module assembly;

5. the focal length $f$;

6. the selection between Wolter-I and Wolter-Schwarzschild geometry; 
7. the approximate mirror module width, $W$. Its value is adjusted from row to row for a) containing an integer number of pores and b) for a petal to include an integer number of modules;

8. the approximate mirror module height, $H$. Its value is tuned for a) containing an integer number of pores and b) an integer number of modules to be included between the minimum and the maximum radius;

9. the radial thickness of structures between consecutive rows;

10. the approximate width of structures between mirror modules;

11. the pore filling factor, $F F \leq 1$ : this limit has been set to avoid obstruction on-axis, i.e., with a smaller pore height than the projected length of the mirror. This parameter is currently expressed via the angular spacing between shells, $\Omega=\Phi-\alpha_{0}^{0} \geq 0$, where $\Phi=h / L_{0}$ is the obstruction parameter and $\alpha_{0}^{0}$ is the on-axis incidence angle on the central plate (Fig. 4).

The program returns a list of the constructive parameter values (number of rows, mirror module width, mirror module length, number of mirror modules in a row, radius of curvature and distance to the focal plane) than can be used to run the effective area simulation code. A variation of some parameters - such as an increase in $w$, or $W$ - exhibits an obvious advantage in terms of optical performances and therefore is not subject to optimization (they clearly have an impact on the mechanical performances, but this issue is not addressed here). Just for example, a layout of an alternative population made of wider modules, and four petals instead of six, is displayed in Fig. 9, right. A computation of the on-axis effective area clearly returns a considerable on-axis EA gain with respect to the standard design, at the expected cost of thinner (and expectedly prone to deform or break more easily) support structures.
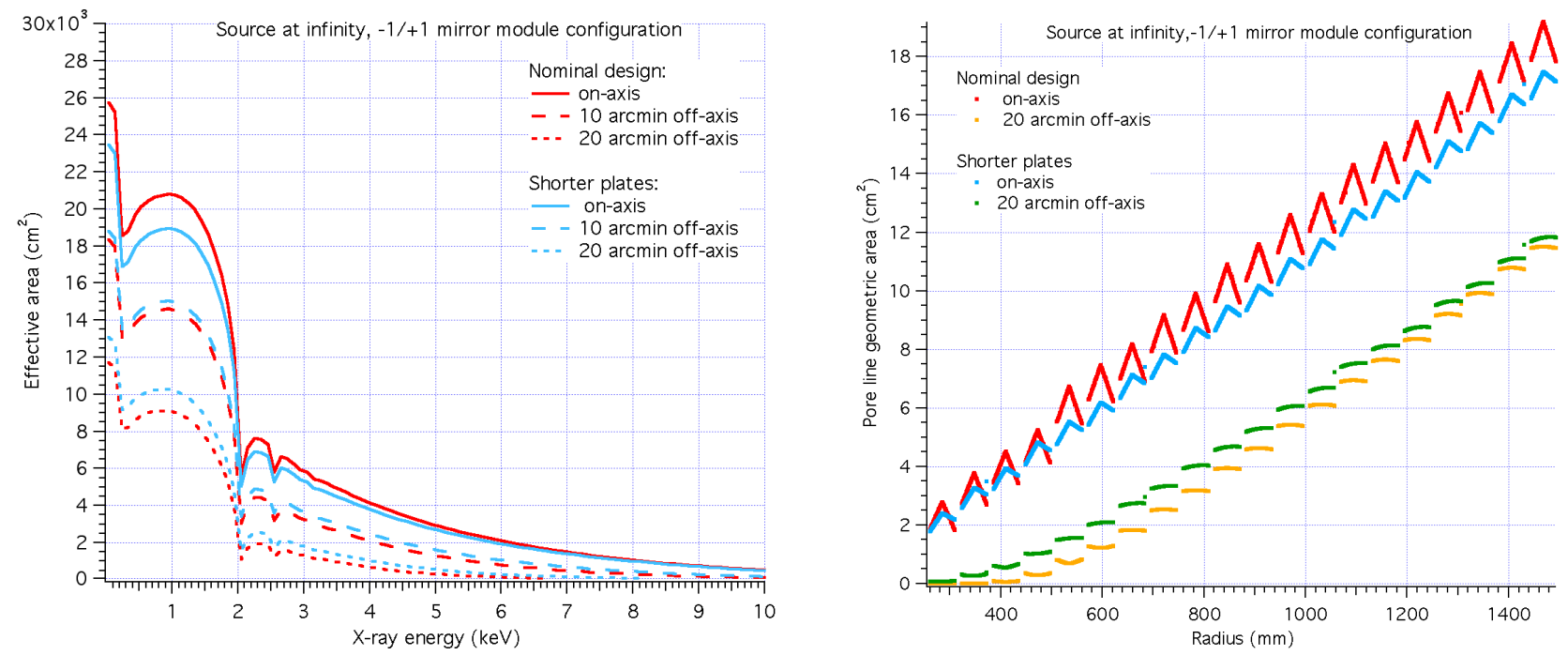

Figure 10. Left: the on-axis area in the nominal design vs. an alternative design with shorter plates. The off-axis EA gain is apparent in the second case. Right: a comparison of the geometric area trends for increasing radius, in the two designs.

The mirror length, $L_{0}$, is a more delicate point as - since $h$ is a constant - it fully determines the filling factor. Its value in each mirror module is variable from row to row and computed as $L_{0}=h /\left(\alpha_{0}^{0}+\Omega\right)$. The standard solution with $\Omega=0$ entails $F F=1$ and is useful to fully exploit the mirror length, but only on-axis. Adopting instead $\Omega>0$ returns shorter $L_{0}$ values: this goes at the expense of the on-axis effective area, but mitigates the off-axis obstruction and enhances the off-axis area. For example, setting $\Omega=10$ arcmin we have obtained an array of plates shorter than in the standard design: we proceeded to compare the EA values in the two cases (Fig. 10, left) on-axis, 10 arcmin, and 20 arcmin off-axis. As expected, the EA is decreased on-axis, but is almost unchanged at $\theta=10 \mathrm{arcmin}$, and enhanced at $\theta=20 \mathrm{arcmin}$. This shows that the field of view 
(FOV) can be enlarged by acting on the plate lengths. A similar behavior is observed in the geometric area trend (Fig. 10, right).

The determination of the plate length using the relation $L_{0}=h /\left(\alpha_{0}^{0}+\Omega\right)$ is quite coarse, however. On one end, $\Omega$ does not correspond exactly to the FOV we are aiming to. In fact, the FOV resulting from this design will turn out to be larger than $2 \Omega$ and will also depend on the incidence angles. This leaves too much room between the mirror walls, which in turn suppresses the self-baffling effect on stray light. Ideally, we should adjust the mirror spacing to the necessary and sufficient value to return exactly the desired FOV: with X-ray optical assemblies made of integral shells, this can be done quite easily. ${ }^{33}$ Unfortunately, this method cannot be immediately applied to pore optics because i) it does not account for the FOV limitation caused by the ribs, ii) the plate spacing $(h+\tau)$ is fixed in SPOs, therefore it is $L_{0}$ that should be varied throughout the stack in order to optimize the off-axis obstruction. Some additional work is needed to adapt this technique to the SPO case.

Another alternative configuration to increase the FOV, without excessively affecting the on-axis EA, is represented by a design with $F F<1$ and $L_{2}>L_{1}$ at the same time. Such a solution is not currently envisaged in the SPO MM production process, but it is definitely worth studying; this interesting configuration will be explored in detail in the next project phase.

\section{RAY-TRACING: MIRROR MODULE MISALIGNMENTS AND STRAY LIGHT}

\subsection{Misalignment simulations using McXtrace and ZEMAX}

Based on the ATHENA standard design, ${ }^{14}$ we have simulated the effect of displacements of SPO MMs on the telescope performance using the ray-tracing software McXtrace. ${ }^{18}$ ATHENA's geometry has been implemented in McXtrace and simulations at pore level have already been reported. ${ }^{34}$ The geometry of each MM varies according to its radial position in the mirror module assembly. In this study, we considered the single SPO MM as a building block and simulated X-rays traveling through one set of stacks (parabolic and hyperbolic) for each row within the ATHENA's mirror assembly. For each of the 20 rows, we ray-traced one set of MMs. The mirror module performance as a function of rigid rotations of the entire MM about the $x, y$, and the $z$-axis is evaluated (we heretofore refer to the frame depicted in Fig. 11), as previously done via a dedicated MATLAB code. ${ }^{13}$ In order to purely assess the effect of displacements, we assumed total reflection for the reflecting coating surface, and only the reflecting surface is considered, i.e., any ray impinging onto the pore sidewalls is absorbed. Finally, we assumed perfect mirrors, i.e., the effect of mirror deformations has not been considered.



Figure 11. The reference frame used in the misalignment/displacement definitions (image credits: Media-Lario).

The effects on the focal spot image aberration, simulated by implementing the proper methods into McXtrace, are shown in Fig. 12. We note that both rotations about the $x$ and the $y$-axis cause a small broadening of the focal spot of each MM, with increasing impact toward the largest radii. The other effect of rotations is the lateral displacement of the focal spot: the magnitude of the effect is depicted in Fig. 13 for the possible rotations around 

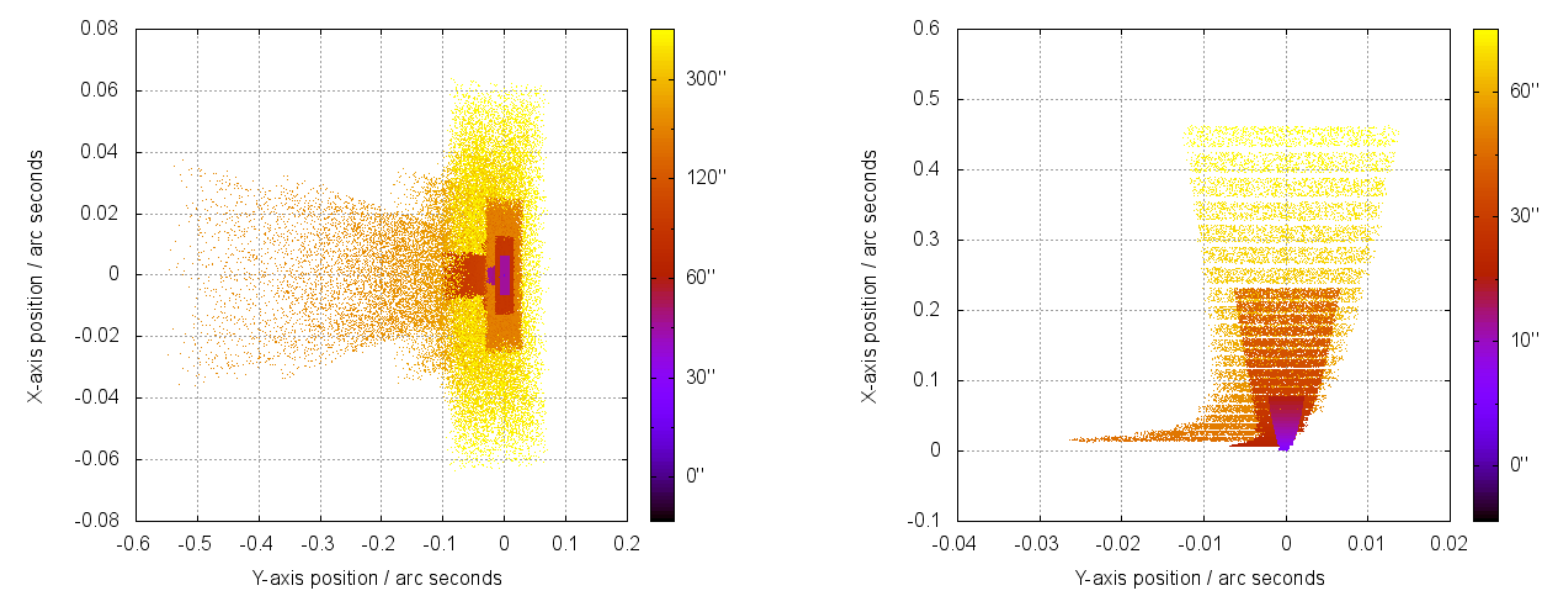

Figure 12. Simulating MM rotations with McXtrace: displacement of the focal spot images with the rotation angles (see the reference frame in Fig. 11). Left: around the $x$-axis (up to 300 arcsec). Right: around the $y$-axis (up to 60 arcsec). Rotations around the $z$-axis do not change the shape of the focal spot.
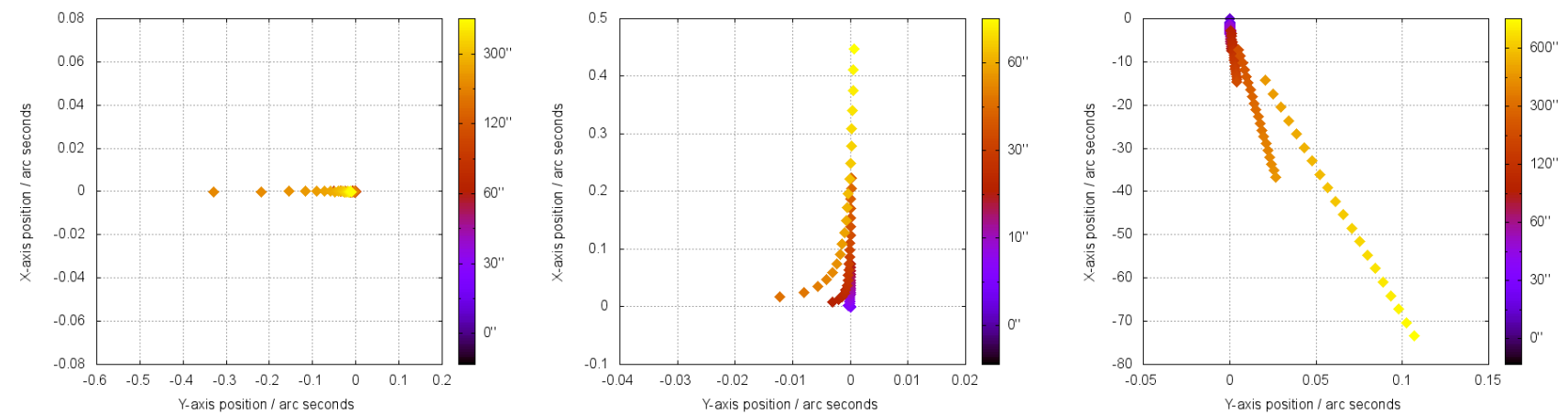

Figure 13. Simulating MM rotations with McXtrace: evolution of the focal spot centroid with the rotation angles. A symbol line represents a succession of MMs in the 20 rows of ATHENA. Left: around the $x$-axis (up to 300 arcsec). Center: around the $y$-axis (up to 60 arcsec). Right: around the $z$-axis (up to 600 arcsec).

the three axes. A rotation around the $x$-axis essentially displaces the image barycenter along the $y$-axis, and vice versa, but the displacements are usually very small as they remain below 0.5 arcsec for quite large misalignments in both directions. Larger displacements occur for MMs at smaller radii in rotations about $x$, and at larger radii around $y$. In contrast, a rotation $\Delta \phi_{z}$ around the $z$-axis does not change the incidence conditions on the mirror surface with respect to the perfect alignment configuration, therefore it does not affect the focal point shape; rather, it displaces laterally the focus centroid by an angle $R_{0} / f \Delta \phi_{z}$. The displacement clearly increases with increasing radius, reaching $\sim 0.12 \Delta \phi_{z}$ in the outermost mirror module row. We can also notice that the results shown here are in perfect quantitative agreement with the ray-tracing simulations previously performed with a dedicated MATLAB code. ${ }^{13}$ This confirms that the methods implemented to simulate the effects of mirror misalignments in McXtrace work correctly.

A further study regards the relative misalignments of the secondary stack with respect to the primary one in each MM, including rotations and translations, which also triggers focal spot aberrations and centroid displacements. This preliminary study was performed implementing a ray-tracing model in the ZEMAX code, in the case of a mirror module located at one of the smallest radii. ${ }^{7}$ In this case, both the intrinsic defocusing of the MM focal spot and the focal spot displacements make the mutual alignment of the stacks critical. In Fig. 14 we show three sets of simulations. aimed at assessing the impact of the three rotations of the secondary stack. The focal spot obtained by tracing a perfectly aligned stack pair is always located in the center of the picture: a small aberration has also been imparted to the mirror profile to return a focal spot in the shape of a small "lily" $(\mathrm{HEW}<1$ arcsec) in order to visualize also the rotation of the entire focus. 
The ZEMAX routine was iterated for a few rotation values about the $x, y$, and $z$-axis, until the focal spot centroid reached the edge of the field in use. As expected, the rotation about the $x$-axis (Fig. 14, left), essentially causes a focal spot displacement along the $y$-axis; as this essentially changes the primary-secondary kink angle, the lateral displacement in angle is four times as large. A rotation around $y$, in contrast, does not change the focal spot centroid: it rather degrades the image stretching the spot along the $y$-axis (Fig. 14, center). Finally, a rotation around $z$ (Fig. 14, right) mostly displaces the focal spot laterally along the $x$-axis: however, the displacement is much less sensitive to rotations around $z$ than around $x$. The analysis will be continued in the next phase of the project, including a statistical distribution of misalignments throughout the MM assembly of ATHENA.


Figure 14. ZEMAX ray-tracing simulations of primary-secondary stack misalignments in a single MM, with $R_{0}=277.5 \mathrm{~mm}$, $W=49 \mathrm{~mm}, L_{0}=110 \mathrm{~mm}, N=34$. The image field is 8.6 arcsec. Left: $0,0.5$, and 1 arcsec rotations about the $x$-axis. Center: a 22 arcsec rotation about the $y$-axis. Right: 0,60, 120, and 180 arcsec rotations about the $z$-axis.

\subsection{Stray light simulation}

A stray light evaluation using McXtrace is - at this stage - performed at pore level, assuming the central pore of each MM as a good representative of all the pore array behavior. The reflecting coating surface is assumed to be the present baseline coating for ATHENA, a bilayer of $\mathrm{Ir} / \mathrm{B}_{4} \mathrm{C}$. Only the coated reflecting surface is considered, while reflections from the silicon pore sidewalls are neglected. We do not consider rays passing through the primary-secondary gaps after the first reflection.
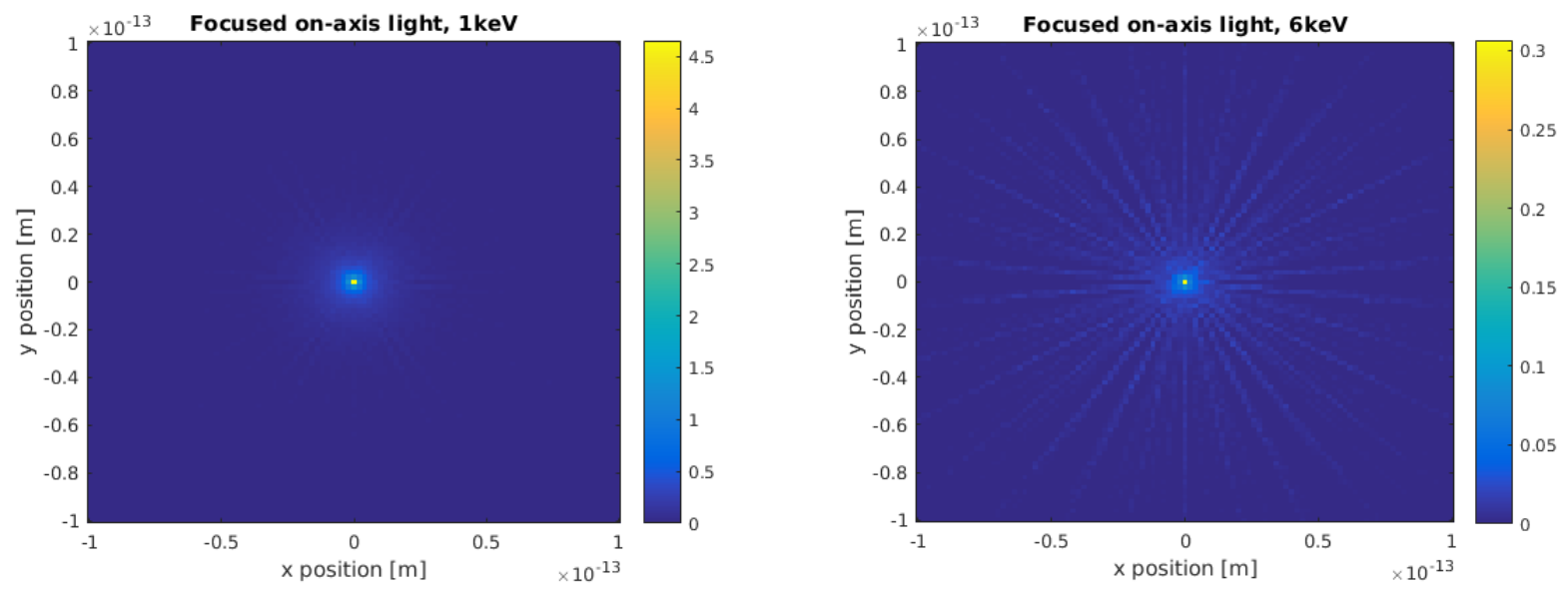

Figure $15.1 \mathrm{keV}$ and $6 \mathrm{keV}$ X-rays focused by all the central pores in the MMs of ATHENA, simulated with McXtrace. The mirrors are assumed as flawless and the source is on-axis, at infinity. The focusing is essentially perfect, as the image side is $2 \times 10^{-13} \mathrm{~m}$. 
The source is unidirectional and planar, initially parallel to the pore pair pupil, and can be rotated with respect to the entire optics array to simulate off-axis angles. Several configurations of the simulation are used to examine different aspects of the optical performance. First, rays from an on-axis, infinitely distant source are sent through all simulated pores (Fig. 15). This serves to evaluate the on-axis focusing ability of the simulated mirrors and so ensure the validity of the simulation. The resulting simulated focal spot is point-like to within a $10^{-13} \mathrm{~m}$ diameter, and would definitely appear as a single pixel of the ATHENA WFI detector. This simulation confirms the numerical accuracy achieved with McXtrace.


Figure 16. Focused X-rays at an off-axis angle of 5 arcmin (top), and an off-axis angle of 30 arcmin (bottom), computed at $1 \mathrm{keV}$ and $6 \mathrm{keV}$. The pixel size is $50 \mu \mathrm{m}$, and the image fields are $2 \mathrm{~mm}$. Off-axis aberrations have now become visible.

The source is then displaced laterally to simulate X-rays incident at various off-axis angles (Fig. 16). Due to the off-axis displacements, variable from module to module (Fig. 13), the circular structure of the optics becomes visible, especially for high off-axis angles, where the pattern grows considerably. Beyond 2 arcmin off-axis, the $1 \mathrm{keV}$ focus is no longer contained within a single $50 \mu \mathrm{m}$ pixel assumed for the simulation. Beyond 5 arcmin off-axis, it is not even contained within a single column of pixels. It should be noted that the pixel size assumed in this simulation - selected to better show the structure of the aberrations - is much smaller than the one foreseen for the WFI (130 $\mu \mathrm{m}$, equivalent to $2.2 \mathrm{arcsec})$. We can also see that there is a clear contrast between the focused photons at $1 \mathrm{keV}$ and $6 \mathrm{keV}$ : for increasing off-axis angles, the incidence angles increase on one side of the mirror assembly, suppressing the reflectivity at higher energies.

We can now apply the McXtrace routine to stray light: rays passing through the pores have been classified in three distinct ways: 1) rays reflected by the parabolic surface, the hyperbolic surface, and focused at the focal point; 2) rays reflected by the parabolic surface and passing directly through the hyperbolic pore without second reflection (P-only rays); 3) rays reflected by the hyperbolic surface without being reflected first on the parabolic 



Figure 17. Focused light and stray light types at the focal plane for off-axis angles of 30, 20, 10, and 5 arcmin, for $6 \mathrm{keV}$ X-rays. The square represents the collecting area of the WFI detector.

pore (H-only rays). The amount of rays that contribute to the stray light, integrated over an infinitely wide focal plane, can be computed easily: $1 \mathrm{keV}$ focused rays suffer from a loss in intensity of about $16 \%$ at 20 arcmin due to stray light, while the $6 \mathrm{keV}$ focused losses in stray light are up to $41 \%$ at 20 arcmin. At $1 \mathrm{keV}$ and $6 \mathrm{keV}$, if the entire focal plane is considered and at so small off-axis angles, $\mathrm{P}$-only rays are the dominant contribution to the stray light. At 30 arcmin, H-only rays emerge significantly, amounting to about $58 \%$ of $6 \mathrm{keV}$ rays, but only $0.64 \%$ of $1 \mathrm{keV}$ rays. The fractions of stray-to-focused light were, however, identified immediately after the optics. This is acceptable for focused rays, because we can always admit all the focused rays to fall within the detector area. In contrast, this is not always true for stray light, which is not focused.

We therefore have to evaluate whether the simulated stray light reaches the detector or not. We assume here the characteristics of the WFI placed at the focal point of ATHENA. We observe that up to 10 arcmin off-axis angle, no stray light reaches the detector area. At $20 \mathrm{arcmin}$, some P-only rays could fall on the corners of the detector, depending on orientation. At 30 arcmin, H-only rays appear on the detector, while the P-only rays recede from the detector. Fig. 17 shows the focused and stray rays at the focal plane for several off-axis angles: beyond 25 arcmin, H-only rays begin to hit the detector, but remains several orders of magnitude fainter than focused light, and could disturb only the observation of very faint objects. The stray light behavior for increasing off-axis angles is a slow increase, followed by an abrupt cutoff. Qualitatively, it resembles the general stray light trend in monolithic X-ray optics, already described analytically. ${ }^{31}$ We will deal with a more exact quantification of the in-detector stray light in the next phase of this project.

\section{SIMULATION OF DIFFRACTIVE EFFECTS}

\subsection{D geometry, double reflection}

In the previous SPIE paper ${ }^{13}$ we started to extend the PSF computation via Fresnel diffraction to the SPO MMs case, accounting for rib structures. More exactly, we reached the conclusion that in the near-field diffraction from the primary to the secondary stacks and for $\lambda$ sufficiently small, the electric field $E_{2}\left(z_{2}\right)$ along a profile $x_{2}\left(z_{2}\right)$ of the secondary pore segment is essentially unaffected by the presence of the ribs. We can therefore go on propagating the electric field to the focal plane (Fig. 18). Doing this, the diffraction pattern can be affected 
by the interference of the pore array, so the diffraction formulae used for conventional X-ray mirrors can be no longer accurate. Therefore, referring to the derivation of the field diffracted by the secondary mirror (Appendix $\mathrm{A}$ in, ${ }^{20}$ with a slight notation change), we can find this expression:

$$
E(x, z)=\frac{\Delta R_{2}}{L_{2} \lambda} \int_{f}^{f+L_{2}} E_{2}\left(z_{2}\right) \frac{x_{2}}{\bar{d}_{2}} e^{-\frac{2 \pi \mathrm{i}}{\lambda} \bar{d}_{2}} \mathrm{~d} z_{2} \int_{-\Delta \Phi / 2}^{+\Delta \Phi / 2} \chi\left(\varphi_{2}\right) e^{-\frac{\pi \mathrm{i} x_{2} x}{\lambda d_{2}} \varphi_{2}^{2}} \mathrm{~d} \varphi_{2},
$$

where $\Delta \Phi$ now denotes the plate azimuthal aperture (for the meaning of the other symbols, we refer to Fig. 18). We consider the mirror reflectivity as a constant throughout this section, and for simplicity we set $r_{\lambda}=1$. To account for the presence of ribs in a plate, we have multiplied the azimuthal integrand into a modulation function $\chi\left(\varphi_{2}\right)$, which equals zero at the polar angles shaded by the ribs, and 1 elsewhere.



Figure 18. Diffraction in a pore optic, reduced to a $1 \mathrm{D}$ geometry. The primary segment initially diffracts the incident wavefront to the secondary segment profile. The subsequent diffraction to the focal plane returns the PSF.

We now introduce the dimensionless parameter $\zeta=\varphi_{2}\left(x x_{2} / \lambda \bar{d}_{2}\right)^{1 / 2}$ and we get

$$
E(x, z)=\frac{\Delta R_{2}}{L_{2} \sqrt{\lambda x}} \int_{f}^{f+L_{2}} E_{2}\left(z_{2}\right) \sqrt{\frac{x_{2}}{\bar{d}_{2}}} e^{-\frac{2 \pi \mathrm{i}}{\lambda} \bar{d}_{2}} \mathrm{~d} z_{2} \int_{-\Delta \zeta / 2}^{+\Delta \zeta / 2} \chi(\zeta) e^{-\mathrm{i} \pi \zeta^{2}} \mathrm{~d} \zeta
$$

where $\Delta \zeta \approx \Delta \Phi\left(R_{0} x / f \lambda\right)^{1 / 2}$. Excepting the case $x=0$, the integration limits are very large as long as $x / f \gg$ $\lambda /(W \Delta \Phi)$. Now, with the test values $R_{0}=1 \mathrm{~m}, W=100 \mathrm{~mm}$, and $\Delta \Phi \approx 0.1 \mathrm{rad}$, we have $x / f \gg 10^{-3}$ arcsec at $\lambda=1 \AA$. Therefore, in X-rays we can very well approximate the integration limits in $\zeta$ with $\pm \infty$. Without the modulation function, the azimuthal integral would simply return a negligible phase factor $\mathrm{e}^{\mathrm{i} \pi / 4}$, so we would find the usual expression of the field at the focal plane, valid for conventional X-ray mirrors, ${ }^{20}$

$$
E(x, z)=\frac{\Delta R_{2}}{L_{2} \sqrt{\lambda x}} \int_{f}^{f+L_{2}} E_{2}\left(z_{2}\right) \sqrt{\frac{x_{2}}{\bar{d}_{2}}} e^{-\frac{2 \pi \mathrm{i}}{\lambda} \bar{d}_{2}} \mathrm{~d} z_{2} .
$$

In UV $(\lambda=1000 \AA)$, we would instead need $x / f \gg 1$ arcsec to justify the approximation $\Delta \zeta \rightarrow \infty$.

When the modulation factor is present, computing analytically the integral in $\zeta$ in Eq. 29 is very difficult, but we can always do it numerically. We show in Fig. 19 the real part of the integral as a function of $x / f$, at $100 \AA$ and $10 \AA$. Without rib modulation (solid lines), both curves exhibit an oscillatory behavior, and eventually converge to 1. in X-rays, however, the integral tends to damp its oscillations rapidly, while in UV light the oscillations persist in a wider angular range. When the $\chi$ function is included (dashed lines), the limit value decreases in both cases. In X-rays, the reduction is $83 \%$, i.e., exactly the reduction expected from geometric optics. We conclude that - at sufficiently small values of $\lambda$ - we can account for the presence of the ribs, also within the 
physical optics framework, by simply re-normalizing the PSF to the obstruction ratio of the plate. These results can be immediately extended to the entire MM, i.e., to a stack of ribbed plates: the optical path difference (OPD) between adjacent plates, observed at an angular distance $\theta$ from the nominal focus, is $h \sin \theta$; to observe any interferential feature, one would need $h \sin \theta \approx \lambda$, i.e., for $\lambda=1 \AA$ and $h=0.7 \mathrm{~mm}$ the first fringe would be seen at $\theta=0.03$ arcsec. This is not detected in practice, because real sources are never perfectly monochromatic (i.e., their coherence length becomes easily longer than the OPD).


Figure 19. The real part of the integral in $\zeta$ in Eq. 29, at (left) $100 \AA$ and (right) $10 \AA$. All the simulations have assumed a $2 \AA$ bandwidth to smooth out sharp interference features, typical of a perfectly monochromatic setup. Dashed lines include a rib modulation with $w+t=1 \mathrm{~mm}$ and $w=0.83 \mathrm{~mm}$ pore widths.

We can conclude that the simulations of SPO MMs in 1D geometry can reliably be performed in X-rays using Eq. 30. This is also confirmed by direct comparison of PSFs computed using both 1D and 2D formalisms (Fig. 25). On the contrary, application of Eq. 30 to the SPO case in UV light can lead to inaccurate results. As we see in the next section, UV simulations are performed more safely in 2D, far-field geometry.

\subsection{D geometry, far-field approximation}

\subsubsection{Experimental validation}

In the case of ATHENA, the long focal length allowed us applying some approximations that reduce the 2D Fresnel integral to a Fourier transform. Since efficient numerical recipes exist to compute the transform (FFT, Fast Fourier Transform), it becomes possible to perform 2D simulations without excessively increasing the computational load. In this approximation we have already shown ${ }^{13}$ that the 2D PSF of an SPO MM at the wavelength $\lambda$, observed at the coordinates $\underline{r}=(x, y)$ on a detector placed at distance $D$ from the intersection plane, can be computed as

$$
\operatorname{PSF}_{\mathrm{M}}^{\mathrm{D}}(\underline{r})=\frac{1}{A_{\mathrm{M}} \lambda^{2} D^{2}}\left|\int_{\mathrm{M}} e^{-\frac{2 \pi \mathrm{i}}{D \lambda} \underline{r}_{1} \cdot \underline{r}} C\left(\underline{r}_{1}\right) \mathrm{d} \underline{r}_{1}^{2}\right|^{2},
$$

where $A_{\mathrm{M}}$ is the aperture area of the MM (including structures), and in which we have denoted the generalized pupil function as

$$
C\left(\underline{r}_{1}\right)=e^{-\frac{\pi \mathrm{i}}{f \lambda}\left[R^{2}+\left(\frac{f}{D}-1\right)\left|\underline{r}_{1}\right|^{2}\right]} \chi_{\mathrm{P}}\left(\underline{r}_{1}\right) \operatorname{CPF}\left(\underline{r}_{1}\right) .
$$

In the $C\left(\underline{r}_{1}\right)$ definition, $f$ is the nominal focal length, $\underline{r}_{1}=\left(x_{1}, y_{1}\right)$ are the coordinates at the MM aperture, $\chi_{\mathrm{P}}\left(\underline{r}_{1}\right)$ is the characteristic function of the MM aperture (equal to 1 where a reflective surface is present at $\underline{r}_{1}$, and 0 elsewhere), $R$ is the intersection plane radius of the primary plate located at $\underline{r}_{1}$, and the complex pupil function

$$
\mathrm{CPF}\left(\underline{r}_{1}\right)=\exp \left[-\frac{2 \pi \mathrm{i}}{\lambda} 2 \varepsilon\left(\underline{r}_{1}\right) \sin \alpha_{R}\right],
$$

accounts for surface errors $\varepsilon\left(\underline{r}_{1}\right)$ projected onto the aperture pupil. Finally, $\alpha_{R}$ is the incidence angle on the plate with radius $R$ : clearly, $R$ and $\alpha_{R}$ take on discrete values. The light source is assumed to be at infinite distance, aligned to the center of the aperture. 

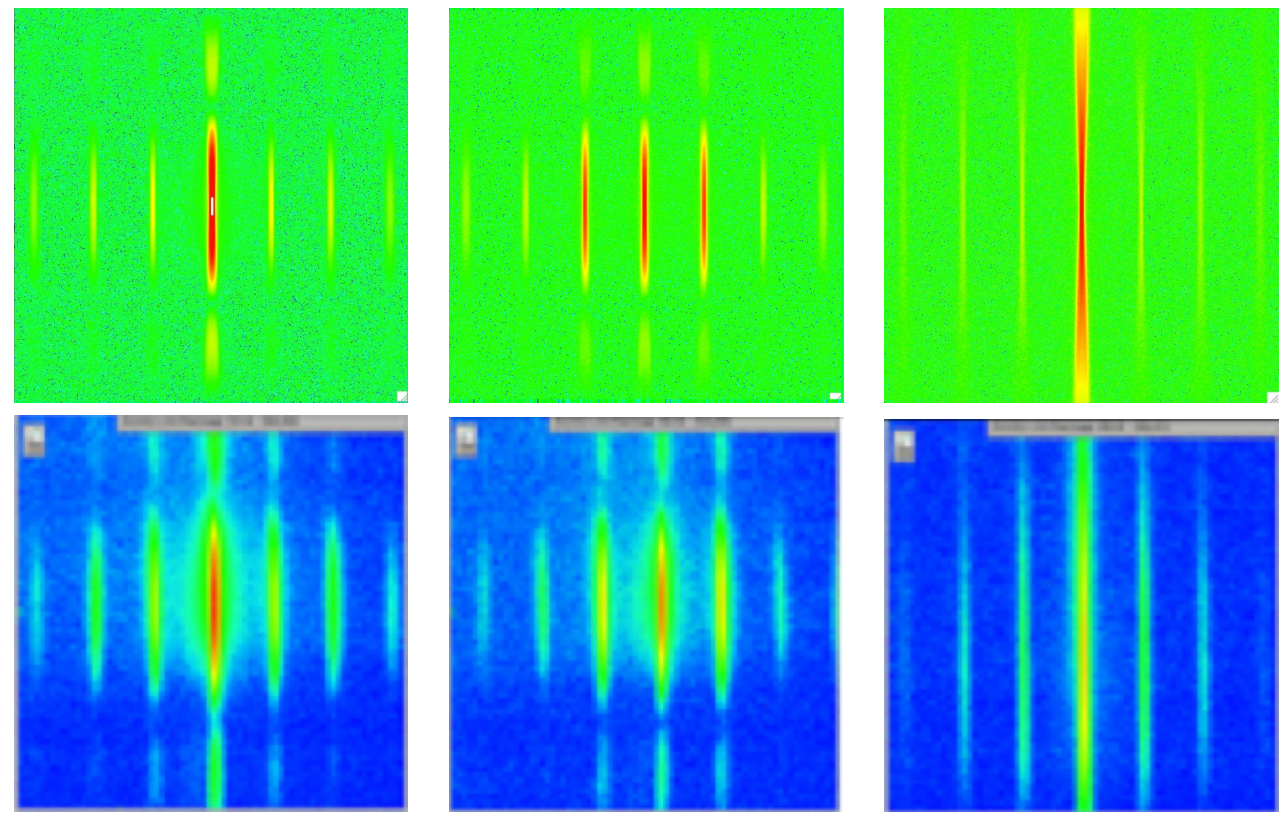

Figure 20. Simulated (top) vs. observed (bottom) diffraction figures in the UV optical bench at Media-Lario $(\lambda=218 \mathrm{~nm}$, $\Delta \lambda=5 \mathrm{~nm}$ ), of an SPO MM provided by ESA. The CCD in use has a $27.6 \mathrm{~mm}$ size and $52 \mu \mathrm{m}$ pixels, the mirror radius is in vertical direction. Left: source on-axis. Center: 11 arcmin off-axis, horizontal rotation in the figure (about the $y$-axis in Fig. 11). The higher orders gain relevance because of the increasing obstruction of ribs, while the pore pitch and the interfringe distance remain constant. Right: 11 arcmin off-axis, vertical rotation in the figure (about the $x$-axis in Fig. 11). The MM tilt caused a reduction of the apparent pore height, but in this case the interference of pore rows is essentially incoherent; hence, the diffraction pattern resembles the one a single pore row.

The computation of the diffraction pattern is therefore reduced to modeling the aperture pupil and implementing Eqs. 31 and 32. The IDL code, which we wrote to this specific aim, takes only 1 to 4 min to complete a PSF computation, using a $2.4 \mathrm{GHz}, 8 \mathrm{~GB}$ RAM MacBook, depending on the MM aperture size and sampling. ${ }^{13}$ We could also provide a successful validation of the code results by direct comparison with experimental diffraction images taken in the Media-Lario optical bench. ${ }^{9}$ The tested mirror module had $f \approx 19 \mathrm{~m}, 34$ plates with $W=L_{0}=65 \mathrm{~mm}, w=0.83 \mathrm{~mm}, h=0.606 \mathrm{~mm}, R_{0}=710 \mathrm{~mm}, t=\tau=0.17 \mathrm{~mm}$. The measurements were performed with a UV collimated source at $\lambda=218 \mathrm{~nm}$, on-axis and off-axis, in the best focus of the MM, and showed a very interesting agreement with simulations (Fig. 20). The finite coherence length of the light was included in the simulated images by repeating the computation at equally-spaced values within a wavelength band $\Delta \lambda=5 \mathrm{~nm}$ centered around $218 \mathrm{~nm}$, and averaging the resulting PSFs. The finite angular size of the source $(4.5$ arcsec) was accounted for by convolving the simulated image with the demagnified source profile. Finally, off-axis cases were also simulated:

i) for a rotation by $\phi_{y}$ about the $y$-axis (Fig. 11), increasing $t$ by $2 L_{0} \phi_{y}$ (Eq. 21) and reducing $w$ by the same quantity (Fig. 20, center);

ii) for a rotation by $\phi_{x}$ about the $x$-axis (Fig. 11), increasing $\tau$ by $2 L_{0} \phi_{x}$ (Eq. 22) and reducing $h$ by the same quantity (Fig. 20, right).

In both cases, a tilt term was added to the OPD maps in order to simulate the lateral displacement of the focal spot (not shown in Fig. 20). In the following sections, we explain how to adapt the formalism to different configurations of the mirror module and of the measurement facility.

\subsection{2 -1/+1 wedge configuration}

In the current standard design, a $-1 /+1$ wedge configuration (Fig. 5) is adopted. In order to simulate the diffraction pattern with this setup, we preliminarily note that the rotation of the reflecting surfaces by $n \Delta \alpha$ causes a negligible PSF degradation, and an effective area loss of just a few percent (as per Eq. 23). The centroid 
position is not changed either. From the viewpoint of the implementation of the $2 \mathrm{D}$ diffraction algorithm, the wedge variation is equivalent to adding two linear phase shifts in the radial direction over each plate, of equal magnitude but opposite signs for the primary and the secondary segment, so in practice they cancel out in the FFT operation. Therefore, Eqs. 31 and 32 can be applied also to this configuration. An accurate definition of the $C\left(\underline{r}_{1}\right)$ requires us to account for the small loss in effective area mentioned above, even with the MM in best align conditions. As we better see in the next section, this can be simply done varying the membrane thickness $\tau$ (neither the rib thickness $t$, nor the focal plane distance $D$, indeed) as if the light source was initially converging to a distance $2 f$ past the module.

\subsubsection{Finite source distance}

The source was hitherto assumed to be at infinity: for an astronomical source, the assumption is certainly correct. It is also correct for the collimated UV light bench at Media-Lario. However, in X-ray calibration facilities (such as BESSY or PANTER) the X-ray beam is located at a finite distance $S$. This causes known effects, such as the displacement of the best focal plane, and the effective area vignetting also on-axis. In this section, we assume the source to be aligned to the MM central pore, i.e., with the incidence angles on the central plate at their nominal value. This is the "calibration mode" $\left(\theta=\delta_{0}\right)$ that minimizes the beam obstruction (Sect. 2.2.2).
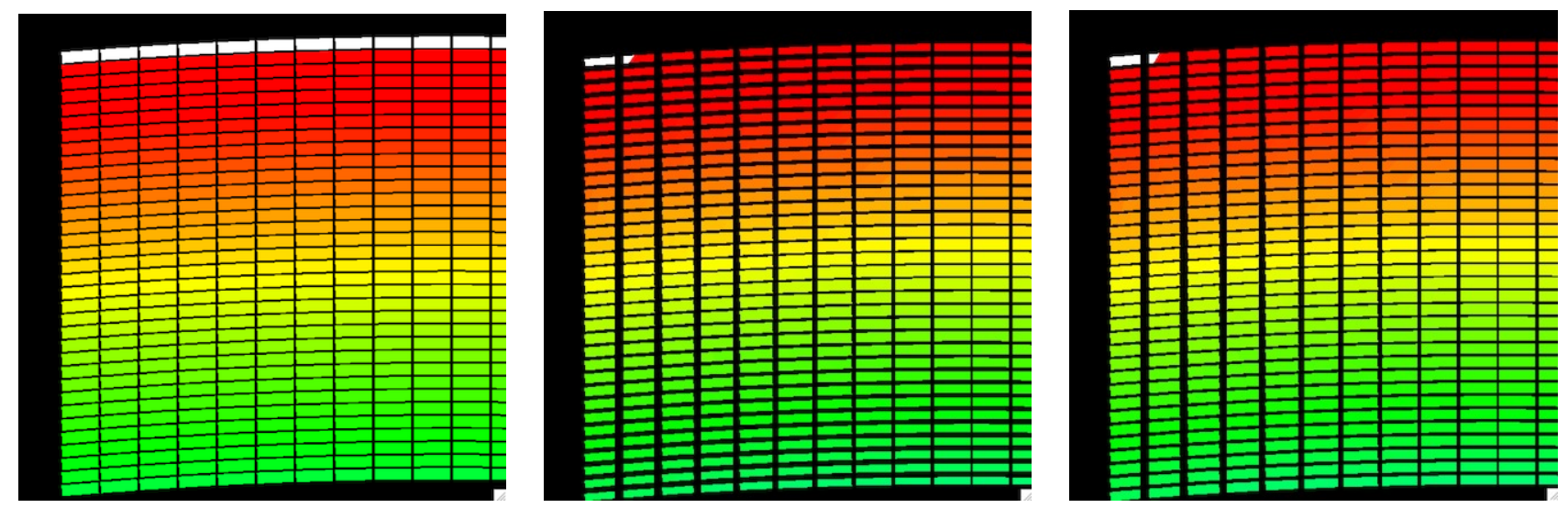

Figure 21. Left: detail of an OPD (optical path difference) map for a MM in $+1 /+3$ wedge configuration, source at infinity on-axis, constant rib thickness, $w=2.1 \mathrm{~mm}, h=0.6 \mathrm{~mm}, f=12 \mathrm{~m}$. In all the pictures, the color variation from green to red denotes increasing OPD throughout the aperture. Black means no optical surface present. Center: the same MM with $S=12 \mathrm{~m}$, aligned to the central pore. The membrane/rib obscuration increases from the center outwards. Right: the same MM with $S=12 \mathrm{~m}$, but in $-1 /+1$ wedge configuration. The membrane vignetting is mitigated, while the rib obscuration is still affected by the finiteness of $S$. The source closeness was deliberately exaggerated in order to highlight the obscuration effect.

To extend the diffraction computation to the finite source distance case, the first thing to do is to include a spherical wave phase term $\pi \mathrm{i}\left|\underline{r}_{1}\right|^{2} / S \lambda$ into the exponent of Eq. 32. Another thing is changing the $\chi_{\mathrm{P}}$ function to $\chi_{\mathrm{P}, S}$, accounting for the increased obstruction of the ribs and the membranes with the source located at distance $S:$ as the divergence angle increases with the residual divergence, the apparent thickness of the structures now increases with the $n, m$ indices in the module (Fig. 3),

$$
\begin{aligned}
t_{m} & =t+2|m| \frac{w+t}{S} L_{0}, \\
w_{m} & =w-2|m| \frac{w+t}{S} L_{0}, \\
\tau_{m} & =\tau+2|n| \frac{h+\tau}{S} L_{0}, \\
h_{m} & =h-2|n| \frac{h+\tau}{S} L_{0} .
\end{aligned}
$$


The generalized CPF therefore becomes

$$
C\left(\underline{r}_{1}\right)=e^{-\frac{\pi \mathrm{i}}{f \lambda}\left[R^{2}+\left(\frac{f}{D}+\frac{f}{S}-1\right)\left|\underline{r}_{1}\right|^{2}\right]} \chi_{\mathrm{P}, S}\left(\underline{r}_{1}\right) \operatorname{CPF}\left(\underline{r}_{1}\right) .
$$

As an example, Fig. 21 shows how the OPD map changes when the source is approached to close distance: as expected, the vignetted regions (in black) become thicker at the expense of the clear pore areas (in color). More exactly, the integral of the colored areas can be computed to return the geometric area of the MM,

$$
A_{\mathrm{G}, S}=\int\left|C\left(\underline{r}_{1}\right)\right| \mathrm{d} \underline{r}_{1}^{2}=\int \chi_{\mathrm{P}, S}^{2} \mathrm{~d} \underline{r}_{1}^{2},
$$

and the values we obtain from Eq. 39 are in very good agreement with those derived via the method described in Sect. 2.1. Using the Parseval theorem, it is also easy to show that the integral of the PSF always equals the obstruction ratio $A_{\mathrm{G}, S} / A_{\mathrm{M}}$. Extension to the off-axis case is straightforward.


Figure 22. Simulated diffraction figures of the SPO MM measured in the UV optical bench (Fig. 20) in X-ray illumination at $\lambda=(40 \pm 0.25) \AA$. Left: source at infinity, $D=f=19 \mathrm{~m}$. The simulated detector has a $2 \mathrm{~mm}$ side and $1 \mu \mathrm{m}$ resolution. Center: simulated within a $6 \mathrm{~mm}$ square area, $S=123 \mathrm{~m}$, and keeping $D$ unchanged: the diffraction pattern is clearly defocused. To ease the comparison with the previous image, the inner frame locates a $2 \mathrm{~mm}$ square. Right: still $S=$ $123 \mathrm{~m}$, after bringing the focal plane to $D=f^{\prime}=22.47 \mathrm{~m}$ (Eq. 40): the best focus position is retrieved.

Once defined $C\left(\underline{r}_{1}\right)$, the PSF of the mirror module is again obtained by application of Eq. 31. The first effect of the finite source distance is a displacement of the best focal plane: therefore, the computation with finite $S$ without adjusting $D$ returns a defocused spot (Fig. 22, center). To retrieve the best focal position, the diffraction figure is to be simulated and observed at $D=f^{\prime}$, given by the usual lens formula,

$$
\frac{1}{f^{\prime}}=\frac{1}{f}-\frac{1}{S}
$$

If this value is substituted into Eq. 38, $C\left(\underline{r}_{1}\right)$ becomes the one of a mirror with focal length $f^{\prime}$, illuminated by a source at infinity and observed in the best focus. The PSF computation (Fig. 22, right) then returns a focal spot that is almost identical to the nominal one. If the MM is in $-1 /+1$ wedge configuration and calibration mode, then the finite distance effects are mitigated in the sole radial direction (Fig. 21, right) by the $n$-th plate pair rotation by an angle $n \Delta \alpha_{\mathrm{w}}$ (Sect. 2.1). In practice, in the best align conditions, the radial vignetting becomes like the source is brought at the effective distance $S^{\prime}$ (see Eq. 27):

$$
\frac{1}{S^{\prime}}=\frac{1}{S}-\frac{1}{2 f},
$$

but the azimuthal vignetting remains unchanged, i.e. it still "sees" the source at a distance $S$. In Fig. 21, for example, we have $f=12 \mathrm{~m}, S=12 \mathrm{~m}$, and $S^{\prime}=24 \mathrm{~m}$. For large $S$, the $S^{\prime}$ value can even become negative, meaning that the radial vignetting behaves as if the beam was converging. In this case, some additional care is needed to properly implement the $C\left(\underline{r}_{1}\right)$ definition into a map to process via FFT. 


\subsubsection{Variable rib spacing}

In the last version of the IDL code for diffraction simulation, particular attention was paid to the radial orientation of ribs. Because all the plates have the same width and the same pore size, the ribs point toward the MM axis but they cannot be exactly stacked radially. This is chiefly evident in a MM near the innermost radius of ATHENA (Fig. 23, left): the alignment of neighboring ribs is gradually lost from the central rib outwards, reducing the rib stacking and affecting the bonding strength of the plates. Therefore, the rib width is increased linearly with the polar angle (Fig. 23, right), improving the rib superposition in neighboring plates. ${ }^{7}$ As the pore width $w$ is kept constant, the pore pitch $w+t$ slightly increases with the polar angle. Once defined the rib thickness variation in the generalized pupil function, the computation of the diffraction pattern is still achieved by applying Eq. 31. In Fig. 24 we show the diffraction figures simulated at $218 \mathrm{~nm}$ for the two mirror modelings.
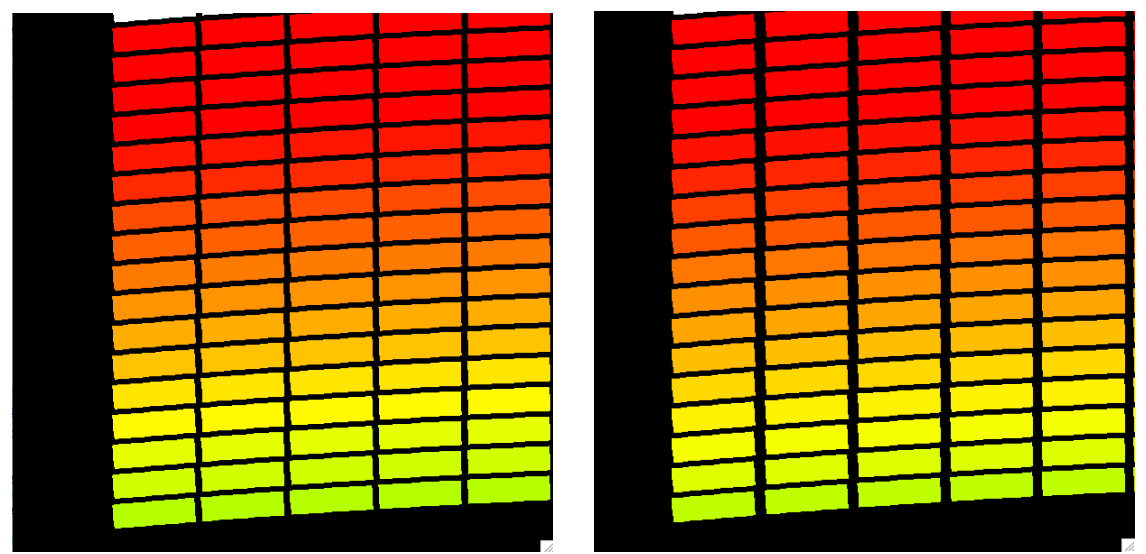

Figure 23. A detail of the OPD map for a MM near the innermost radius of ATHENA. Left: constant rib thickness. Right: variable rib thickness with the lateral coordinate. The MM parameter values ${ }^{7}$ are: $f=12 \mathrm{~m}, R_{0}=277 \mathrm{~mm}, N=34, L_{0}$ $=110 \mathrm{~mm}, h=0.6 \mathrm{~mm}, \tau=0.14 \mathrm{~mm}, w=2.1 \mathrm{~mm}, t=0.17 \mathrm{~mm}$ at the center rib and (in the right graph) increasing by $0.013 \mathrm{~mm} /$ pore from the central ribs toward the plate edges.
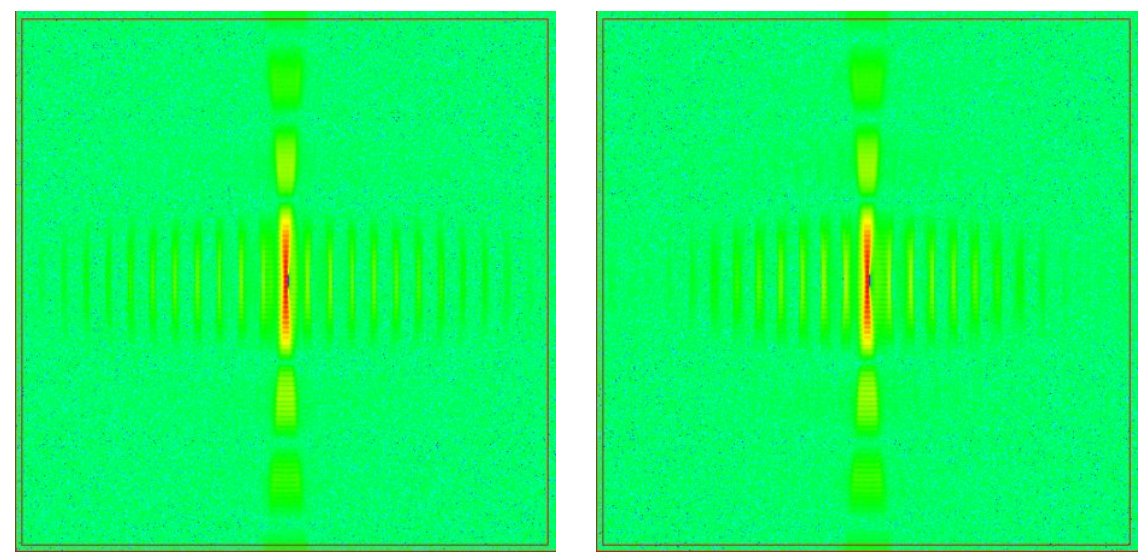

Figure 24. Simulated diffraction figures of the mirror modules shown in Fig. 23, at 218 nm. Left: constant rib thickness. Right: variable rib thickness. Minor differences can be detected in the intensity of the secondary maxima.

\subsubsection{Simulating imperfect SPO surfaces}

The diffraction figures shown so far were the ones of perfect mirror modules. The diffraction pattern of an imperfect SPO MM can be simulated projecting the modeled or measured figure error of the primary or secondary plates onto the MM aperture. In this way, we obtain a $\varepsilon\left(\underline{r}_{1}\right)$ error map, which we can substitute into Eq. 33 . To this end, if $\Delta r_{1}$ is the aperture pupil sampling step ${ }^{13}$ then the mirror profile error map has to be resampled at $\Delta r_{1} / \sin \alpha_{R}$ along the $n$-th plate longitudinal direction (that becomes the radial direction in the $C\left(\underline{r}_{1}\right)$ map), and 
at a $\Delta r_{1}$ step in the azimuthal direction. Consequently, the longitudinal error scale of the errors is compressed by a factor $\sin \alpha_{R}$ with respect to the real profile, while the azimuthal scale remains unchanged. Hence, the longitudinal slopes are enhanced by a factor $1 / \sin \alpha_{R}$ with respect to the azimuthal ones; we can thereby expect - as per geometric optics - beam deflections in the radial direction $1 / \sin \alpha_{R}$ larger than along the azimuth, even if the two errors have the same amplitude (then attenuated by a $\sin \alpha_{R}$ factor in the exponent of Eq. 33).

The PSF simply follows by application of Eqs. 31 and 32, where the CPF term has now become a complex function provided by Eq. 33. For example, assuming that all the pores in the MM have the same profile error, $\varepsilon\left(\underline{r}_{1}\right)$ can be modeled analytically or resampled numerically to be a periodic function along the radius with period $h+\tau$, and along the plate width with period $w+t$. In Fig. 25 we show the simulation of $0.3 \mathrm{keV}$ PSFs for a single stack mirror module with $f=12 \mathrm{~m}, W=65 \mathrm{~mm}, R_{0}=750 \mathrm{~mm}, w=0.83 \mathrm{~mm}, h=0.6 \mathrm{~mm}, t=\tau=$ $0.17 \mathrm{~mm}$. Different longitudinal profile errors have been assumed as $\varepsilon\left(\underline{r}_{1}\right)$, either sinusoids with variable periods and phases, or peculiar deformations mostly affecting the profile edges. Several profile errors were selected to be asymmetric in order to displace the PSF centroid to positive $y$ values. This allowed us to assess the accurate correspondence of the centroid measured in UV illumination with the expected one in X-rays (Fig. 26).
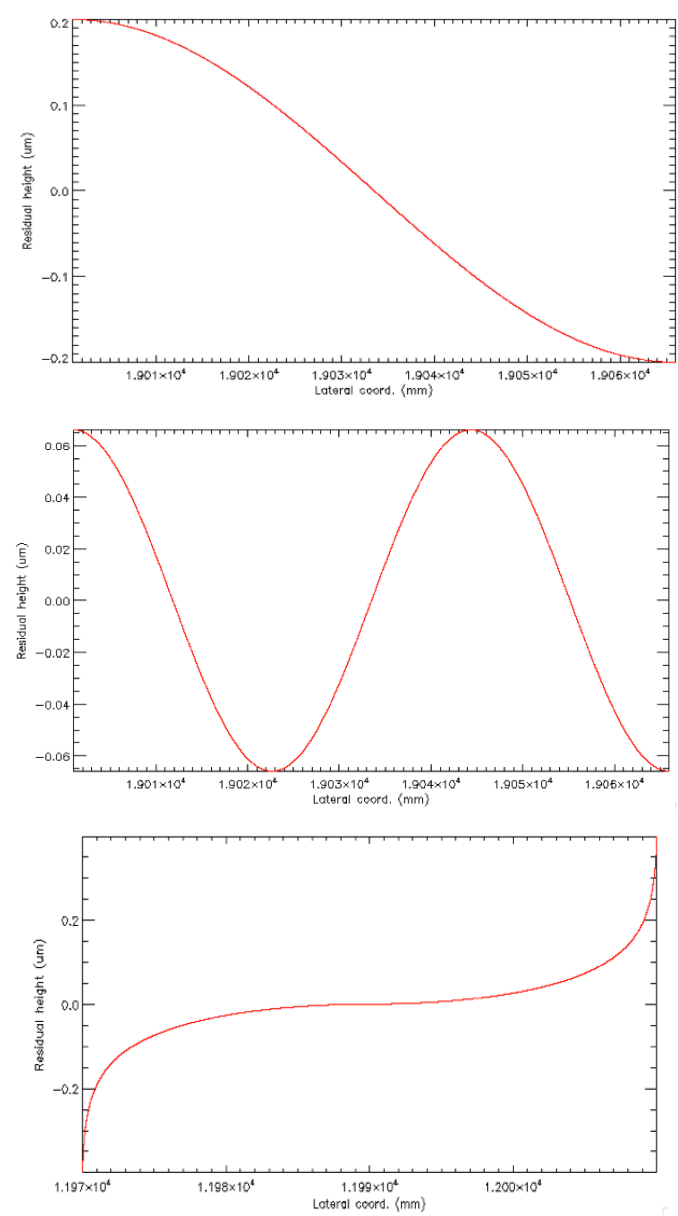
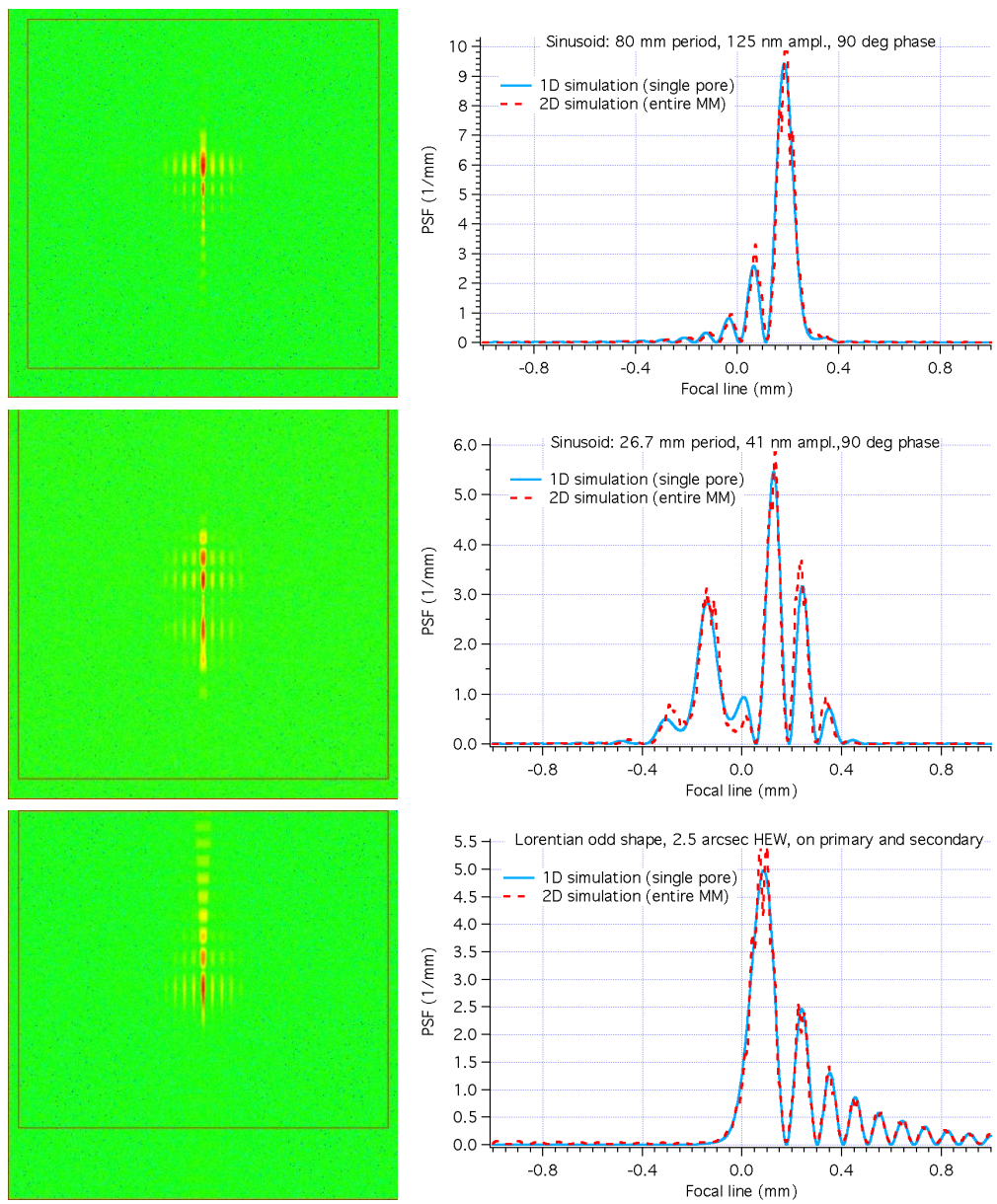

Figure 25. Left: longitudinal profile perturbations imparted to the primary or the secondary segment of an SPO MM. Center: the 2D diffraction pattern computed at $(40 \pm 0.25) \AA$, within a $2 \mathrm{~mm}$ field. Right: comparison between the $2 \mathrm{D}$ PSF (dashed lines), derived from diffraction patterns, and the 1D PSD (solid lines), computed from the exact double diffraction using a profile pair. The perturbation in the bottom line is the odd version of a profile tailored to return a Lorentzian PSF. ${ }^{35}$

We could also check the accuracy of the simulated PSFs by comparison with 1D simulations, obtained applying Eq. 30 to the longitudinal perturbations shown in Fig. 25, left. Although applied to a single profile pair, the resulting 1D PSFs in the X-ray band are in perfect accord with the 2D simulation findings from the entire MM 
(Fig. 25, right). In UV light, however, the interference of pores yields non-negligible effects and the same 1D-2D agreement would be not this accurate. This confirms the conclusions reached in Sect. 4.1.

Finally, we have repeated the 2D computations of Fig. 25 in UV light $(218 \mathrm{~nm})$, simulating in this way the PSFs expected in the Media-Lario optical bench (Sect. 4.2.1), with the same profile errors. Visually, the PSF does not change significantly when figure errors are added to the simulation, being dominated by the aperture diffraction term. However, the computed centroid does move, also in the UV simulation, to a $y$ coordinate that depends on the profile error adopted and that, moreover, accurately follows the centroid displacement at $0.3 \mathrm{keV}$ (Fig. 26, left). In addition to the experimental proof reported in another paper of this volume, ${ }^{9}$ this simulation demonstrates the reliability of the optical alignment in a UV bench at locating the expected position of the focal spot in X-rays, in order to co-focally integrate the ATHENA optical assembly. We can also prove theoretically the centroid invariance with $\lambda$, but we postpone the proof to a subsequent work.

Finally, we also report an example of 2D diffraction pattern, simulated supposing an azimuthal profile error of the plates (Fig. 26, right). As expected, their impact is much smaller than the longitudinal ones, therefore very ample azimuthal errors (100 $\mu \mathrm{m}$ peak-to-valley or more) have to be modeled to see relevant effects on the PSF.
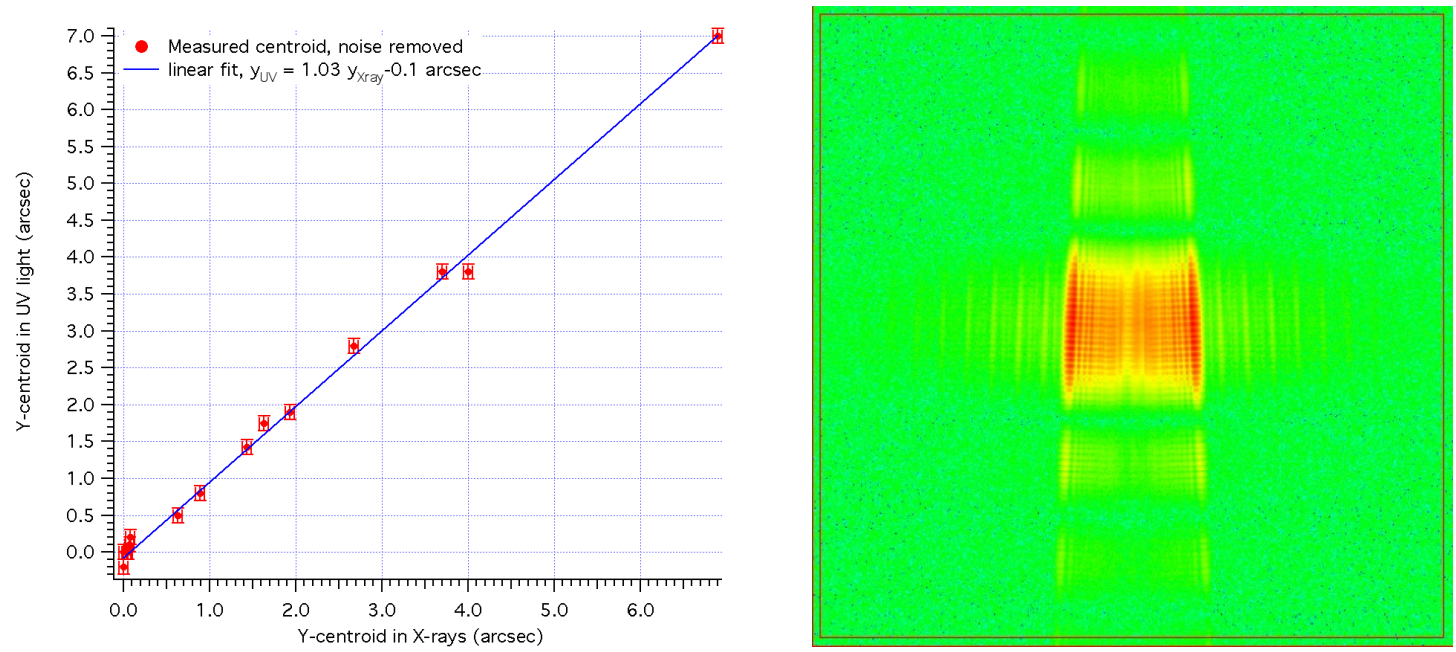

Figure 26. Left: comparison of the centroid displacements expected in X-rays (abscissae) vs. the centroid coordinate computed in UV light (ordinates). The deviation from the quadrant bisector does not exceed, excepting only one case, 0.2 arcsec. Right: a computed diffraction pattern of the mirror module in UV light, after adding a transversal figure error with co-sinusoidal shape, $2 W$ period, and $150 \mu \mathrm{m}$ amplitude.

\section{SURFACE ROUGHNESS AND SCATTERING LEVEL ASSESSMENT}

Being a surface diffraction effect, the X-rays scattering from surface roughness can in principle be simulated using the 2D theory (Sect. 4.2.5). From the surface PSD (power-spectral-density) characterization, it is possible to generate roughness maps compatible with that PSD and add them to the error map $\varepsilon\left(\underline{r}_{1}\right)$ of SPO plates: the PSF naturally follows from the application of the far-field theory (Eq. 31). Even if this procedure has been successfully tested with near-normal incidence mirrors, ${ }^{36}$ in grazing incidence the longitudinal scale of roughness - typically in the micron range - would be compressed by a $\alpha_{R}$ factor, making the aperture pupil sampling very tight. This would in turn increase the size of the matrix to be processed via FFT, and probably the computation time, beyond acceptable limits.

The evaluation of the roughness impact is easier in 1D geometry, using 1D Fresnel diffraction tools ${ }^{20}$ or, in the smooth-surface approximation - widely applicable to the SPO MM cases - the first-order scattering theory. We have made use of the latter in last year's work, ${ }^{13}$ when we had analyzed X-ray scattering test data on multilayer-coated SPO samples and compared the results with surface roughness PSDs measured at OAB (using the MFT phase shift interferometer) and at DTU (via AFM, atomic force microscope), finding a very interesting 
agreement among all the techniques used (Fig. 28, right). We had also found that the expected HEW degradation due to surface roughness did not exceed 3.5 arcsec at $6 \mathrm{keV}$, to be added linearly to the 5 arcsec HEW tolerance at $1 \mathrm{keV}$, where the roughness has expectedly a negligible impact (Fig. 29, left). The total HEW of 8.5 arcsec fitted within the 10 arcsec HEW tolerance at $6 \mathrm{keV}$.

However, that evaluation did not consider two aspects: 1) the HEW evolution modeling used the outer roughness of the coating as input data, and did not include the PSD evolution throughout the stack; 2) the measured sample did not undergo the photolithography process needed to keep the rib locations coating-free and so ensure the stacked plate adhesion. The lift-off phase typically leaves residues on the optical surface of the plates, ${ }^{22}$ thereby affecting the substrate smoothness and the one of the reflective coating that will be deposited subsequently. In this work, we have investigated these two aspects by direct PSD measurement of a set of SPO plates provided by DTU in different phases of the coating deposition: with/without patterning with photoresist stripes, and before/after multilayer deposition. From the PSD comparison, it is possible to notice the roughness alteration caused by the photoresist residuals and by the coating deposition.
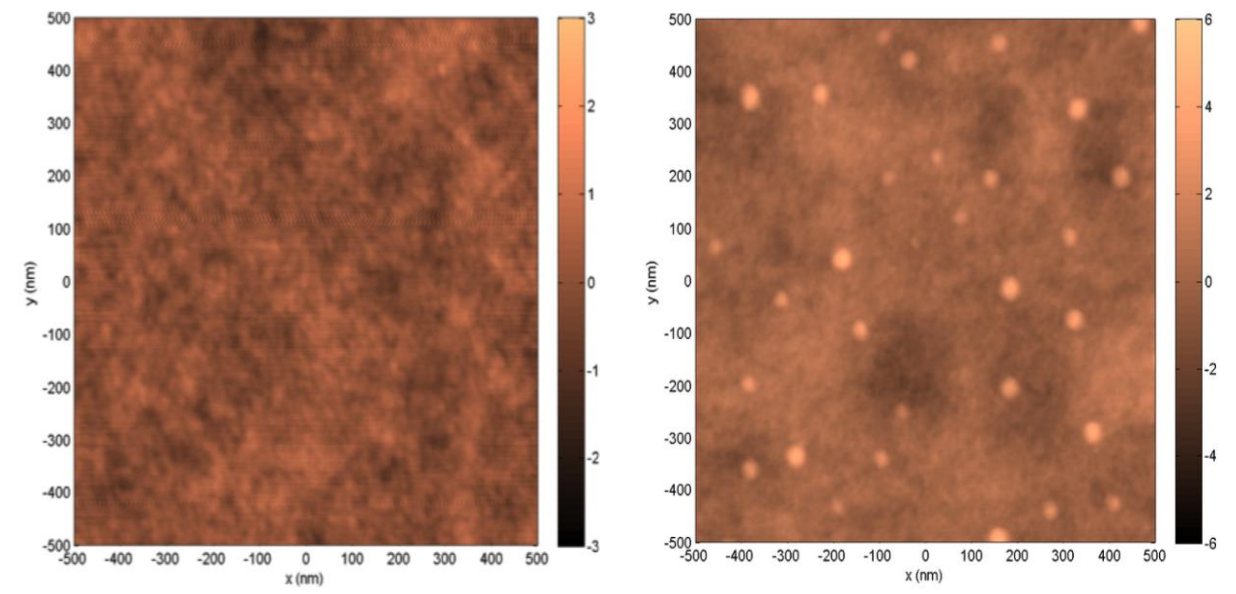

Figure 27. AFM maps of ribbed silicon plates, over $1 \mu \mathrm{m}$ range. Left: without photoresist application. Right: with photoresist deposition, patterning, and liftoff.

New surface topography measurements of samples before multilayer coating showed that photoresist residues are present on the silicon plates after rib patterning on the optical side. The residues appear like islands with sub-micron diameter and a few nm height (Fig. 27): their presence increases the surface roughness from approx. $3 \AA$ without residues to approx. $6 \AA$ in a typical $1 \mu \mathrm{m}$ scan. However, the PSD degradation is not limited to a spatial wavelengths window near $1 \mu \mathrm{m}$ : their spatial distribution affects the PSD up to several tens of microns. This can be seen not only via AFM imaging but also in the MFT measurements (Fig. 28, left).

As for the PSD evolution throughout the multilayer coating, measurements performed before and after coating deposition showed that the coating leaves the roughness essentially unchanged in the $1 \mathrm{~mm}-10 \mu \mathrm{m}$ range of spatial wavelengths, the one mostly affecting the scattering at X-ray energies below $10 \mathrm{keV}$, while roughness is even smoothed out by the coating in the spectral band $10 \mu \mathrm{m}-0.5 \mu \mathrm{m}$ rather than being degraded (Fig. 28, right). Moreover, the multilayer seems to have a higher smoothing efficiency than the bilayer. As a matter of fact, relaxation of the surface topography at high-spatial frequencies can be sometimes observed ${ }^{37}$ in high-quality thin films deposited by magnetron sputtering. A similar smoothing effect was observed with the coating deposited on the patterned plate, on which measurement were taken at the locations between ribs, but the coating influence is insufficient to balance the effect of photoresist contaminations. Anyway, the PSD evolution through the stack seems to be quite small. This explains the accord between the surface data and the unique PSD used to model the $6 \mathrm{keV}$ X-ray scattering scans taken at BESSY on the multilayer-coated sample, ${ }^{13}$ without evolution assumptions (we also have to consider that the reflection at $6 \mathrm{keV}$ chiefly occurred at the first two $\mathrm{Ir} / \mathrm{B}_{4} \mathrm{C}$ interfaces in that multilayer; therefore, the scattering diagram is only minimally affected by the roughness of the buried layers).

We now pass to the assessment of the X-ray scattering effects: if the PSD evolution in the coating can be neglected, we have to evaluate the impact of photoresist contaminations. A quick computation of the scattering 

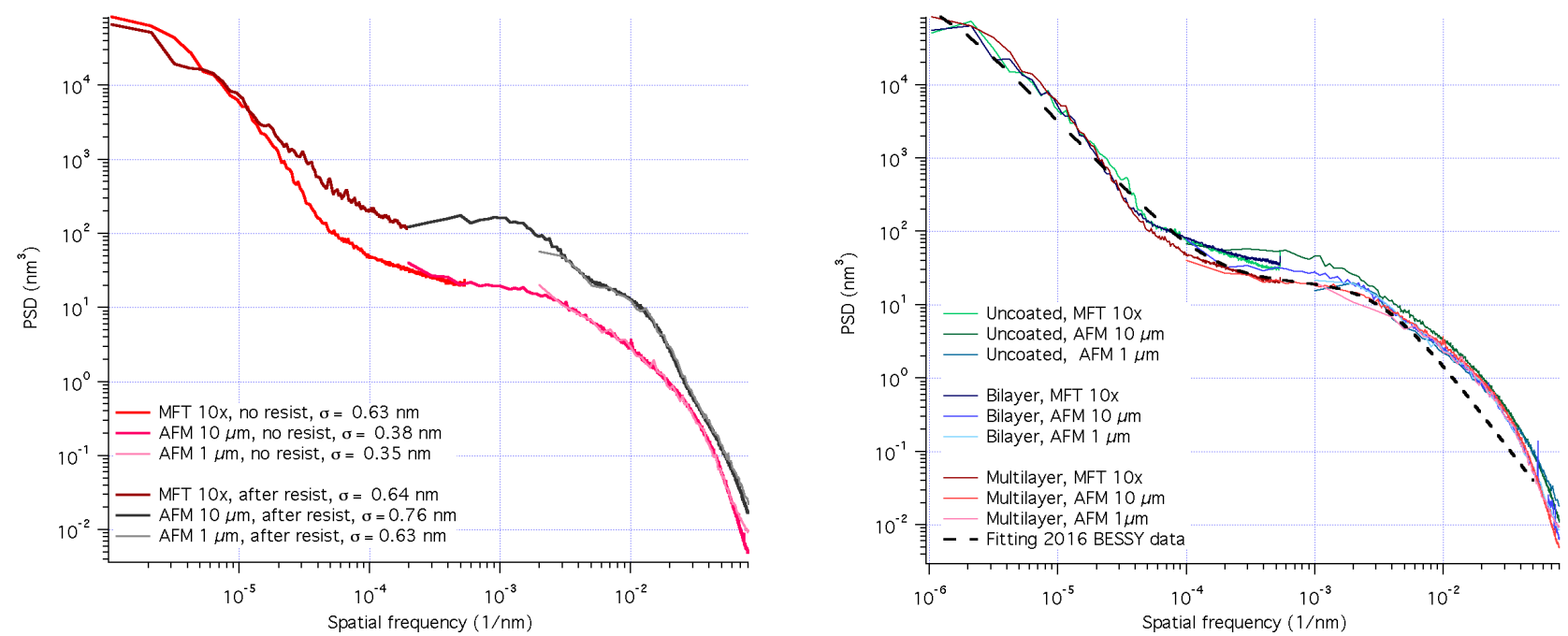

Figure 28. PSD analysis using the MFT $10 \times$ and the AFM. Left: on samples with vs. without photoresist residues, after multilayer deposition. Right: on samples without resist residues, before (green) and after bilayer (blue) or multilayer (red) deposition.

diagram using the PSDs reported in Fig. 28 shows that we may expect no visible effect by the residues at $1 \mathrm{keV}$, while some enhancement starts to become visible in the scattering wings from $5 \mathrm{keV}$ on. To make the analysis quantitative, we have repeated the computation of the expected HEW trend with increasing energy from a PSD ${ }^{21}$ this time using the PSD with contaminations. The cumulative curves for increasing number of mirror module rows are shown in Fig. 29, right, still assuming a 5 arcsec HEW at low energies to be added linearly to the scattering term. Comparison with the corresponding curves (Fig. 29, left) without residues ${ }^{13}$ shows that the scattering effect of photoresist particles starts to become visible at $5 \mathrm{keV}$. At $6 \mathrm{keV}$, the expected HEW is 9.5 arcsec, 1 arcsec more than the value predicted for a contamination-free surface and on the edge of the tolerable 10 arcsec for ATHENA at that energy. However, this leaves no margins for other possible degradation factors of the HEW or the EA; therefore, a new lift-off process is being developed by Cosine and DTU in order to return residue-free patterned plates.
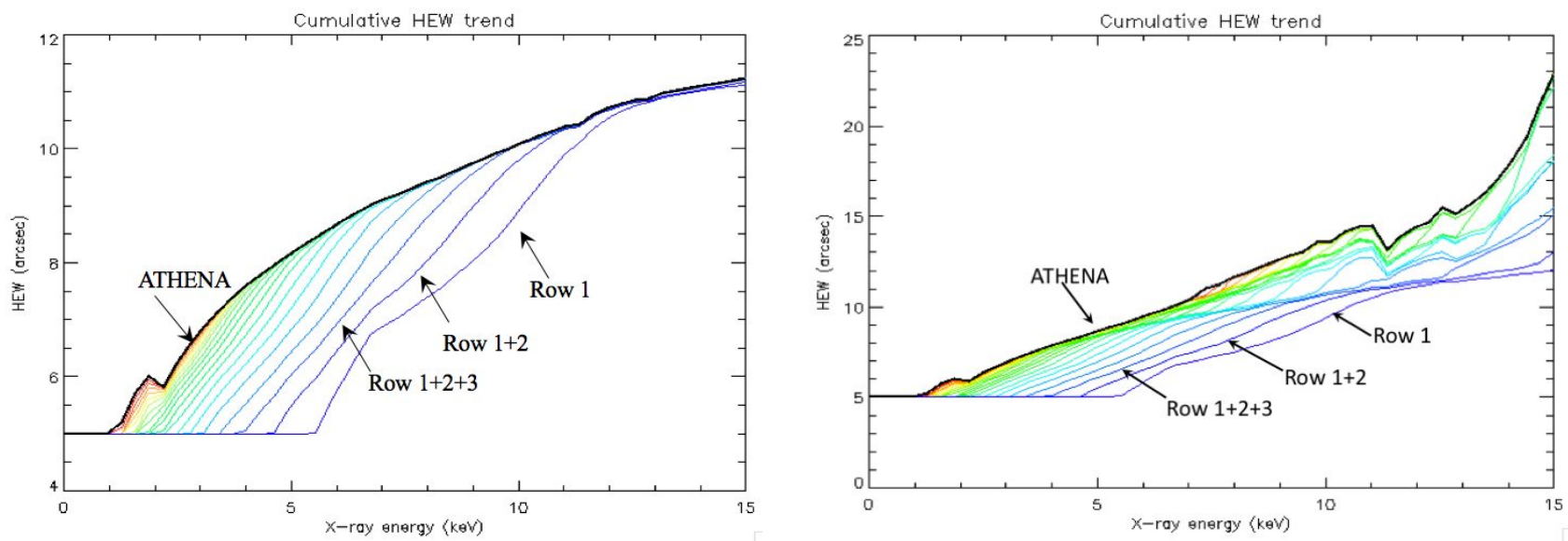

Figure 29. The computed HEW as a function of the X-ray energy for the entire ATHENA telescope (black line), assuming the standard $\mathrm{Ir} / \mathrm{B}_{4} \mathrm{C}$ multilayer coating and the PSDs shown in Fig. 28, left. The colored lines are the cumulated contributions of increasing radii from blue to red. Left: using the PSD without photoresist residues. Right: using the PSD with photoresist residues, the scattering contribution is higher from $5 \mathrm{keV}$ on (notice the vertical scale change). 


\section{MAGNETIC DIVERTER SIMULATIONS: THE HALBACH ARRAY}

As a follow-up of last year's work, we also have developed simulations for a magnetic diverter (MD) onboard ATHENA, aiming at reducing the proton and electron flux contributing to the total background. This year, we have simulated the Halbach array solution, an arrangement of permanent magnets in which the magnetic dipole moment changes its orientation in order to provide an uni-directional magnetic flux in the surrounding space. Initially developed for synchrotron radiation undulators as a linear array, it is also designed in a ring-like shape in order to return an intense magnetic field inside the bore with minimal escape field outside the ring. This would be a suitable geometry for ATHENA, because focused X-rays propagate unperturbed in the bore, while charged particles collected by the optics are deflected laterally.

Due to the difficult manufacturing of magnetic rings with a continuously-varying magnetic dipole orientation, a closed Halbach array design is usually approximated as an assembly of trapezoidal magnets with appropriate orientation of the magnetization.* Our implementation into an IDL simulation code (Fig. 30, left) consists of 8 magnetic bars with constant magnetization magnitude and variable direction with the polar angle, plus 16 wedged magnets with triangular bases to complete the gaps. The mid-diameter of the array is $60 \mathrm{~cm}$, and the magnets enclose a $50 \mathrm{~cm}$ bore diameter in order to keep the field of the WFI completely unobstructed. The vertical thickness of the magnets can be varied to find the best tradeoff between total mass and deflection efficiency. For the rectangular bars, the magnetic field expression is the same $u_{s e d}{ }^{28}$ and detailed ${ }^{29}$ in previous works. As for the wedges, the magnetic field dependence on the local coordinates is reported in another document. ${ }^{30}$ The total magnetic field is obtained by field superposition from the elements composing the array.

For example, assuming a $1.3 \mathrm{~T}$ magnetic remanence ( $\mathrm{NdFeB}$, N52 grade) and a $6 \mathrm{~cm}$ magnet thickness, we have drawn some $\underline{B}$ field lines in Fig. 30, right: within the bore, they are mostly oriented along the $y$-axis. the $\underline{B}$ magnitude is is $500-1000 \mathrm{G}$ in a wide region near the center of the bore, while the residual field at the focal plane has a magnitude of $0.17 \mathrm{G}$. This is an acceptable magnetic field $(<1 \mathrm{G})$ in order to not disturb the proper working of the detectors. Assuming a typical density of $7.5 \mathrm{~g} / \mathrm{cm}^{3}$ for NdFeB sintered magnets, we can estimate a total mass of $90 \mathrm{~kg}$ for such a magnetic assembly.
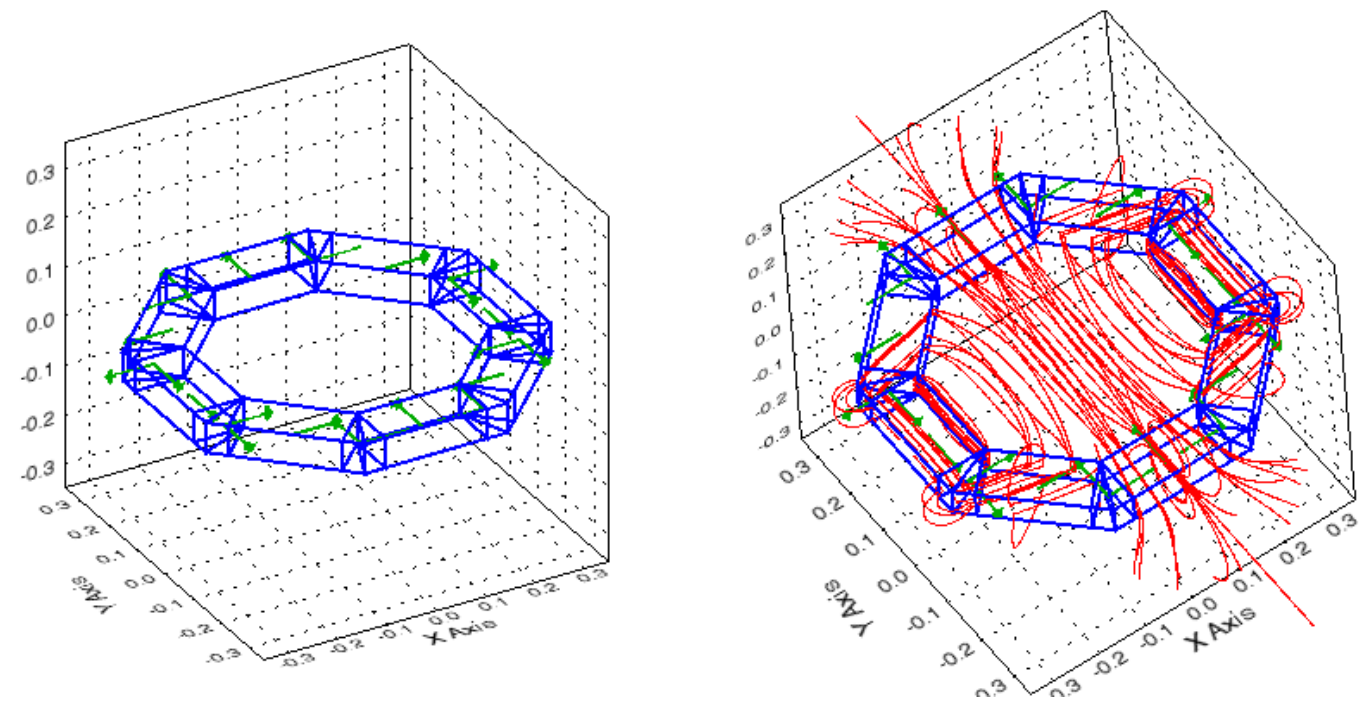

Figure 30. Left: general layout of the Halbach array, modeled as an assembly of bars and wedges. In this figure, the magnets are $6 \mathrm{~cm}$ thick. The green arrows indicate the orientation of the magnetization vectors. Right: simulated magnetic induction field lines in the array mid-plane.

The magnetic field generated by the array has been tested by simulating a beam of soft protons up to an energy threshold of $70 \mathrm{keV}$. Accounting for the energy loss in optical filters (especially those of X-IFU), the events generated in the detectors by such protons would have a $20 \mathrm{keV}$ maximum kinetic energy. ${ }^{23}$ Higher energy events would definitely be recognized as charged particles and rejected. The deflection of the proton

\footnotetext{
${ }^{*}$ Find more details at https://en.wikipedia.org/wiki/Halbach_array
} 
trajectories by the MD is visibly effective, at least using a perfectly collimated beam (Fig. 31, left): a realistic beam reflected/scattered by the optics in grazing incidence reflection is, however, characterized by a relevant dispersion in directions. We have thereby simulated the behavior of a beam with Gaussian dispersion in the angle formed by the velocities with the $z$-axis $(2 \mathrm{deg} \mathrm{rms})$ and uniform dispersion in the transverse angle. As the beam dispersion was originated at optics level, the proton beam had much room to diverge and reached the MD much broader than shown in Fig. 31, so most protons fell outside the bore and were rejected. The particles that passed through the MD were instead deflected laterally by the magnetic field. On the other hand, electrons with the same energetic and angular distribution were completely repelled by the intense magnetic field, already in the MD vicinities (Fig. 31, right). Even if electrons experience a different attenuation in the detector filters, and therefore the initial population that might generate background event has potentially much higher kinetic energies, their angular deflection in the MD will expectedly be large enough to make them miss the WFI detector (and, a fortiori, the X-IFU).


Figure 31. Initially collimated particle fluxes, simulated while passing through the Halbach array shown in Fig. 30. The colors change from green to red with increasing kinetic energies of the particles, from 1 to $70 \mathrm{keV}$. Left: protons are deflected laterally. Right: electrons are repelled.
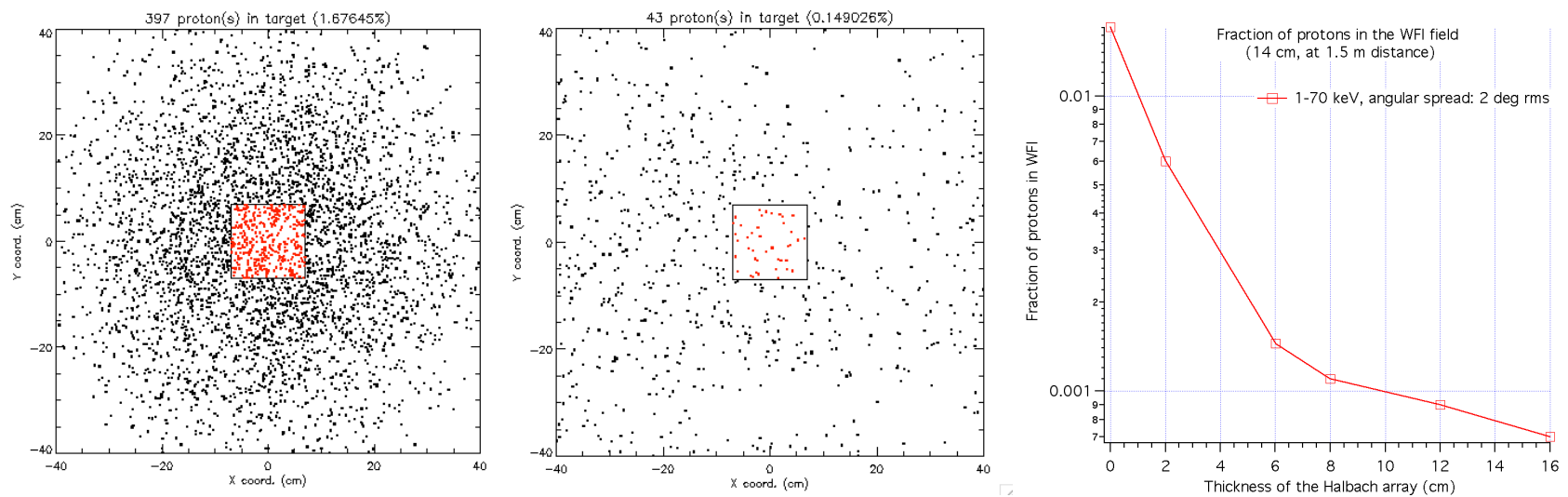

Figure 32. Results of the 30000-particle tracing, assuming a Gaussian angular distribution with 2 deg rms in the axial angle. The central square represents the WFI area from which proton trajectories should be deviated. Left: without magnetic field. Center: with magnetic field: the particle cloud core has moved to the right side, out of the graph. Right: improvement of the MD performances for increasing magnet thickness in the Halbach array.

We could run detailed simulations launching 30000 protons with kinetic energies uniformly distributed between 1 and $70 \mathrm{keV}$. Extending the trajectories of the protons to the focal plane, we could evaluate the fraction of charged particles that fell within the WFI (a squared area with a $14 \mathrm{~cm}$ side, centered in the focal plane). Two typical images of the focal plane are shown in Fig. 32, without (left) and with Halbach array (center). The 
comparison clearly shows that the insertion of a Halbach array with $6 \mathrm{~cm}$ thick magnets causes a reduction of the proton dose by a 11-fold factor. We have also repeated the simulation varying the thickness values of the MD, keeping all the other parameters unchanged. The fraction of protons in the WFI area is reported in Fig. 32, right: increasing the thickness, the MD leakage ratio falls exponentially up to a $6 \mathrm{~cm}$ thickness, then it starts to decrease much less rapidly.

For completeness, we have also evaluated the forces and the torques acting on some magnetic pieces in their nominal positions. The force computation is not implemented yet for magnetic wedges, so the magnets under test are hereby approximated by rectangular bars, and the following values should be considered lower limits. Anyway, while the torques exerted on the magnets are negligible, the forces are quite intense. For example, the magnets parallel to the $y$-axis in Fig. 30 are repelled outwards by a $>365 \mathrm{~N}$ force, mostly directed along the $x$-axis. In contrast, the two magnets parallel to the $x$-axis are attracted toward the center of the array by a $>$ $330 \mathrm{~N}$ force. Finally, the tilted magnets are even subject to a $>634 \mathrm{~N}$ force outwards along $x$, and to an almost equal force inwards along $y$. Shortly put, the array tends to collapse along $y$ and to disrupt itself along $x$ : if a Halbach array is implemented in ATHENA, robust structures will have to be foreseen to keep the magnets in place!

\section{CONCLUSIONS}

A set of simulation and modeling tools are being developed in a collaboration between institutes involved in the ATHENA project, covering crucial topics of the SPO-based design of the ATHENA optics, including: analytical design, ray-tracing predictions, diffractive effect simulations, particle tracing, and scattering level assessment from surface metrology. These activities will be further developed in the next year to support the design, the performance simulation, the realization, the integration, the alignment, and the verification of the ATHENA optical mirror assembly.

\section{ACKNOWLEDGMENTS}

This work is supported by ESA (contracts No.: 4000114410 SIMPOSiuM, 4000114931 ASPHEA, 4000111244 SPIRIT). We thank all the AREMBES collaboration for the profitable discussions on the MD topic.

\section{REFERENCES}

[1] Bavdaz, M., Wille, E., Shortt, B., Fransen, S., et al., "The ATHENA optics development," Proc. SPIE 9905, $990525(2016)$

[2] Collon, M., Vacanti, G., Günther, R., et al., "Silicon pore optics for the ATHENA telescope," Proc. SPIE 9905, $990528(2016)$

[3] Della Monica Ferreira, D., Massahi, S., Christensen, F., et al., "Design, development, and performance of x-ray mirror coatings for the Athena mission," Proc. SPIE 10399, 1039918 (2017)

[4] Van Speybroeck, L., Chase, R., "Design parameters of paraboloid-hyperboloid telescopes for X-ray astronomy," Appl. Opt. 11(2), 440 (1972)

[5] Massahi, S., Girou, D., Della Monica Ferreira, D., et al., "Investigation of photolithography process on SPOs for the ATHENA mission," Proc. SPIE 9603, 96030M (2015)

[6] Bavdaz, M., Wille, E., Ayre, M., et al., "The Athena telescope and optics status," Proc. SPIE 10399, 103990B (2017)

[7] Wille, E., Bavdaz, M., Oosterbroek, T., et al., "Silicon Pore Optics Mirror Modules for Inner and Outer Radii," Proc. SPIE 9603, 96030L (2015)

[8] Collon, M., Vacanti, G., Barrière, N., et al., "Development of Athena mirror modules," Proc. SPIE 10399, 103990C (2017)

[9] Valsecchi, G., Marioni, F., Bianucci, G., et al., "Optical integration of SPO mirror modules in the ATHENA telescope," Proc. SPIE 10399, 103990E (2017)

[10] Perinati, E., Diebold, S., Guzman, A., et al., "Background studies for ATHENA: status of the activities at IAAT," Proc. SPIE 9905, 990565 (2016)

[11] Lotti, S., Macculi, C., D'Andrea, M., et al., "Updates on the background estimates for the X-IFU instrument onboard of the ATHENA mission," Proc. SPIE 9905, 990563 (2016) 
[12] Burwitz, V., "Testing and calibrating the Athena optics at PANTER," Proc. SPIE 10399, this conference

[13] Spiga, D., Christensen, F., Bavdaz, M., et al, "Simulation and modeling of silicon pore optics for the ATHENA X-ray telescope," Proc. SPIE 9905, 96055 O (2016)

[14] Oosterbroek, T., "ATHENA telescope reference design and effective area estimates," ESA document SREF/2014-033 (2014)

[15] Conconi, P., Campana, S., "Optimization of grazing incidence mirrors and its application to wide-field X-ray telescopes," A\&A 372, 1088-1094 (2001)

[16] Spiga, D., Cotroneo, V., Basso, S., Conconi, P., "Analytical computation of the off-axis effective area of grazing incidence X-ray mirrors," A\&A 505(1), 373 (2009)

[17] Spiga, D., "Optics for X-ray telescopes: analytical treatment of the off-axis effective area of mirrors in optical modules," A\&A 529, A18 (2011)

[18] Bergbäck Knudsen, E., Prodi, A., Baltser, J., et al., "McXtrace: a Monte Carlo software package for simulating X-ray optics, beamlines and experiments," Journal of Applied Crystallography 46, 679-696 (2013)

[19] Spiga, D., Raimondi, L., "X-ray optical systems: from metrology to Point Spread Function," Proc. SPIE 9209, 92090E (2014)

[20] Raimondi, L., Spiga, D., "Mirrors for X-ray telescopes: Fresnel diffraction-based computation of point spread functions from metrology," A\&A 573, A22 (2015)

[21] Spiga, D., "Analytical evaluation of the X-ray scattering contribution to imaging degradation in grazingincidence X-ray telescopes," A\&A 468, 775 (2007)

[22] Massahi, S., Della Monica Ferreira, D., Christensen, F., Shortt, B., et al., "Development and production of a multilayer-coated x-ray reflecting stack for the Athena mission," Proc. SPIE 9905, 99055P (2016)

[23] Molendi, S., Gastaldello, F., Rossetti, M., Spiga, D., Tiengo, A., "A quick and not entirely clean estimate of the Soft Proton impact on Athena," INAF-IASF technical note (2015)

[24] Dichter, B., Woolf, S., "Grazing angle proton scattering: effects on Chandra and XMM-Newton X-ray telescopes," IEEE Trans. Nucl. Sci. 50(6) 2292 (2003)

[25] Firsov, O., "Reflection of fast ions from a dense medium at glancing angles," Sov. Phys. Doclady 11(1), 732 (1967)

[26] Diebold, S., Tenzer, C., Perinati, E., et al., "Soft Proton Scattering Efficiency Measurements on X-Ray Mirror Shells," Exp. Astr. 39(2), 343 (2015)

[27] Fioretti, V., Bulgarelli, A., Malaguti, G., Spiga, D., Tiengo, A., "Monte Carlo simulations of soft proton flares: testing the physics with XMM-Newton," Proc. SPIE 9905, 99056U (2016)

[28] Spiga, D., Fioretti, V., Bulgarelli, A., Dell'Orto, E., et al., "A magnetic diverter for charged particle background rejection in the SIMBOL-X telescope," Proc. SPIE 7011, 70112Y (2008)

[29] Spiga, D., Mattaini, E. , Parodi, G., Ottolina, M., Fioretti, V., Tiengo, A., "Notes on the development of a magnetic diverter for the SIMBOL-X X-ray telescope," INAF/OAB int. rep. 06/2009 (2009)

[30] Spiga, D., "Charged particle diverters for X-ray telescopes: the magnetic field of a uniformly-magnetized wedge," INAF/OAB int. rep. 07/2017 (2017)

[31] Spiga, D., "Analytical computation of stray light in nested mirror modules for x-ray telescopes," Proc. SPIE 9603, 96030H (2015)

[32] Spiga, D., "X-ray mirror module design from exact FOV requirement: extension to the case of on-axis obstructed mirrors," INAF/OAB int. rep. 12/2016 (2016)

[33] Spiga, D., "X-ray mirror module analytical design from field of view requirement and stray light tolerances," Proc. SPIE 9905, 99056R (2016)

[34] Della Monica Ferreira, D., Bergbäck Knudsen, E., Westergaard, N., et al., "Simulating X-ray telescopes with McXtrace: A case study of ATHENA's optics," Proc. SPIE 9905, 990576 (2016)

[35] Spiga, D. , Raimondi, L., Svetina, C., Zangrando, M., "X-ray beam-shaping via deformable mirrors: Analytical computation of the required mirror profile," NIM-A 710, 125 (2013)

[36] Tayabaly, K., Spiga, D., Sironi, G., et al., "Point spread function computation in normal incidence for rough optical surfaces," Proc. SPIE 9911, 99111X (2016)

[37] Canestrari, R., Spiga, D., Pareschi, G., "Analysis of microroughness evolution in X-ray astronomical multilayer mirrors by surface topography with the MPES program and by X-ray scattering," Proc. SPIE 6266 , $626613(2006)$ 